\title{
Shear instability of plastically-deforming metals in high-velocity impact welding
}

\author{
Ali Nassiri a,b,", Brad Kinsey ${ }^{a}$, Greg Chini ${ }^{\text {a,b }}$
}

\section{Abstract}

High-speed oblique impact of two metal plates results in the development of an intense shear region at their interface leading to interfacial profile distortion and interatomic bonding. If the relative velocity is sufficient, a distinct wavy morphology with a well-defined amplitude and wavelength is observed. Emergence of this morphology below the melting point of the metal plates is usually taken as evidence of a successful weld. Among various proposed mechanisms, instability owing to large tangential velocity variations near the interface has received significant attention. With one exception, the few quantitative stability analyses of this proposed mechanism have treated an anti-symmetric/shear-layer base profile (i.e., a KelvinHelmholtz configuration) and employed an inviscid or Newtonian viscous fluid constitutive relation. The former stipulation implies the energy source for the instability is the presumed relative shearing motion of the two plates, while the latter is appropriate only if melting occurs locally near the interface. In this study, these restrictions, which are at odds with the conditions realized in high-velocity impact welding, are relaxed. A quantitative temporal linear stability analysis is performed to investigate whether the interfacial wave morphology could be the signature of a shear-driven high strain-rate instability of a perfectly plastic material undergoing a jet-like deformation near the interface. The resulting partial differential eigenvalue problem is solved numerically using a spectral collocation method in which customized boundary conditions near the interface are implemented to properly treat the singularity arising from the vanishing of the base flow strain-rate at the symmetry plane of the jet. The solution of the eigenvalue problem yields the wavelength and growth rate of the dominant wave-like

a Department of Mechanical Engineering, University of New Hampshire, 33 Academic Way, Durham, NH 03824, USA

b Program in Integrated Applied Mathematics, University of New Hampshire, 33 Academic Way, Durham, NH 03824, USA

* Corresponding author. Tel./Fax: +1-603-862-2156

E-mail address: ali.nassiri@unh.edu 
disturbances along the interface and confirms that a shear instability of a plastically-deforming material is compatible with the emergent wavy interfacial morphology.

Keywords: Stability analysis, interfacial waves, high-velocity impact welding, perfectly-plastic material, singular eigenvalue problem

\section{Introduction}

In the automotive, aerospace, appliance, and various other industries, there is a primary need for lightweight structures. This goal can be achieved by using multi-material assemblies, but owing to the disparate melting temperatures of the materials, traditional fusion welding processes cannot be used. One of the most remarkable ways to join dissimilar metals is by High Velocity Impact Welding (HVIW). As depicted in Fig. 1, HVIW encompasses a class of methods including EXplosive Welding (EXW), Magnetic Pulsed Welding (MPW), Laser Impact Welding (LIW), and Vaporizing Foil Actuator Welding (VFAW). High precision, short cycle time, less wrinkling and uniform strain distribution are among the outstanding advantages of HVIW.

In all these methods, the metals are joined by first accelerating a flier workpiece (i.e., either a metal tube or a metal plate) to a high velocity with a significant kinetic energy before it strikes a stationary workpiece. The methods differ in the precise mechanism by which the flier workpiece is accelerated. In EXW, a chemical explosive creates a shock wave in the fluid medium to accelerate the flier workpiece. In MPW, the flier workpiece is accelerated by a repulsive magnetic field and Lorentz forces. In LIW, laser-generated optical energy is converted to kinetic energy through ablation at the surface and confinement of the gas generated between the flier and base plates. In VFAW, the flier is launched toward the base plate by the pressure deficit created from the electrically-driven rapid vaporization of a thin metallic conductor. In addition to the differences in the acceleration mechanism, the length scale of the weld also differs across these methods. For example, EXW is usually applied for large-scale operations with weld lengths on the order of meters and thicknesses on the order of centimeters. MPW and VFAW are suitable for smaller-scale operations with weld lengths on the order of centimeters to millimeters and thicknesses on the order of millimeters [1]. LIW is used for components with thicknesses ranging from millimeters to microns. Clearly, HVIW is a multidisciplinary research area involving the dynamics of collisions at high velocities and 
pressures and the transient fluid-like thermo-mechanical behavior of metals at high strain rates and temperatures.

a)

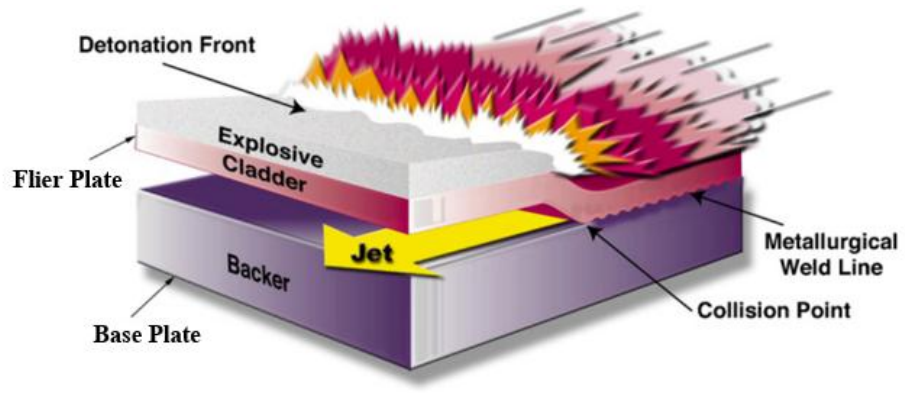

b)
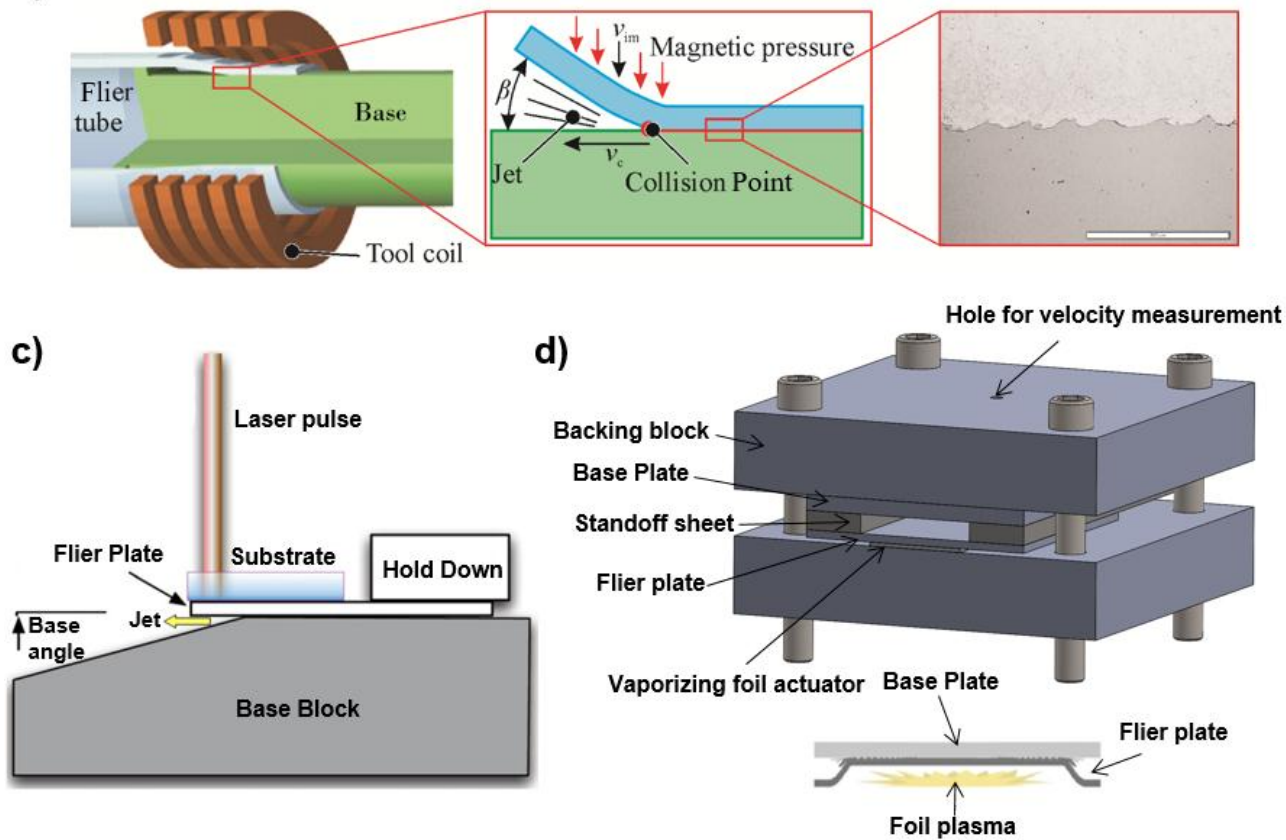

Fig. 1. Schematic of High Velocity Impact Welding (HVIW) methods a) EXplosive Welding (EXW) [2], b) Magnetic Pulsed Welding (MPW) [3] , c) Laser Impact Welding (LIW) [2], and Vaporizing Foil Actuator Welding (VFAW) [1]

A striking feature of metals joined using HVIW is the emergence of a characteristic wavy morphology at the interface between the two welded workpieces. This distinctive signature, with its well-defined amplitude and wavelength, is observed in all the variants of HVIW provided that the velocity of the flier workpiece is sufficient [4,5] (e.g., $>250 \mathrm{~m} / \mathrm{s})$; see Fig. 2 . Emergence of this wavy pattern at temperatures below the melting point of the metal plates is generally taken as evidence of a successful weld. 

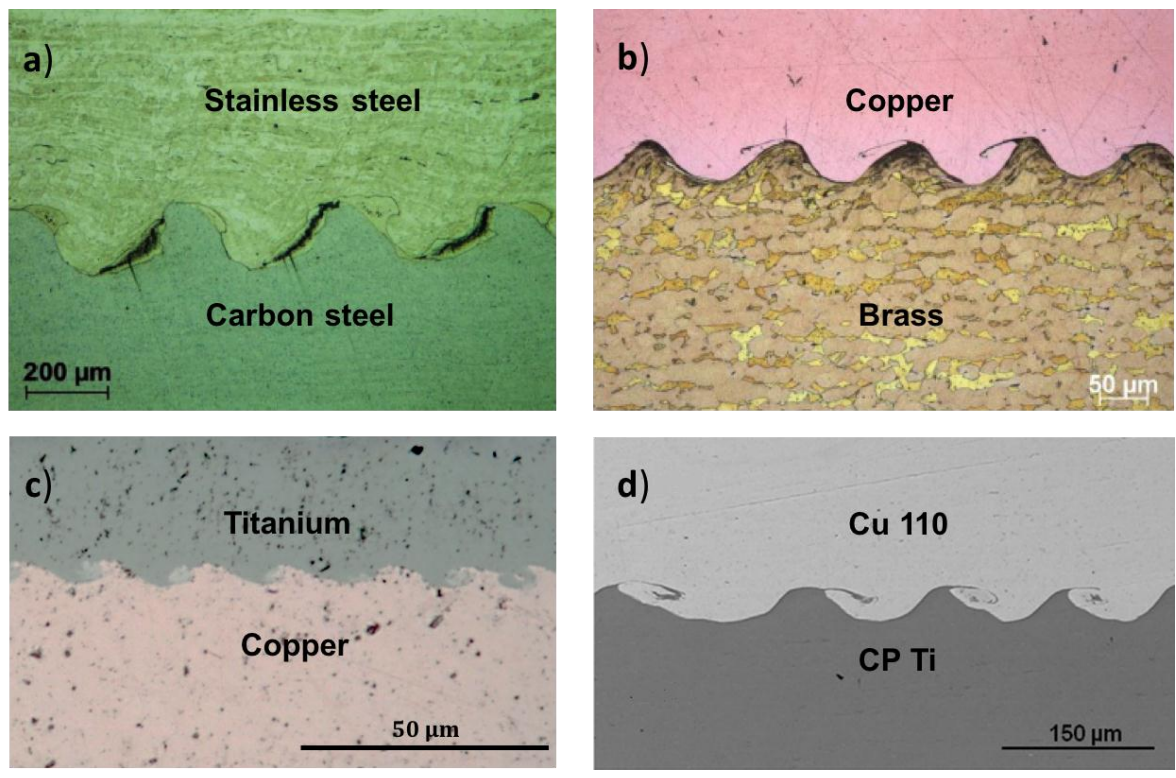

Fig. 2. Wavy morphology observed during a) EXW [6] , b) MPW [7], C) LIW [8], and c) VFAW [9]

The specific physical mechanism that gives rise to the wavy interfacial morphology has been the subject of many research investigations over the past five decades yet remains incompletely understood. Previously proposed wave formation theories can be grouped into one of the following categories [10] : the jet indentation mechanism [11,12], the stress wave mechanism [13] , the vortex shedding mechanism [14] , and the shear instability mechanism [15-17]. As the pioneers of the first category (i.e., the jet indentation mechanism), Abrahamson [11] and Bahrani et al. [12] conducted experimental tests with jets impinging on grease and silicon putty and also with Nickel/Copper explosive welding. They described how the interface could obtain its characteristic shape by periodic indentation of the base plate and hump formation ahead of the stagnation point by the flier plate and vice versa (see Fig. 3). Abrahamson [11] was the first to propose that the formation of a re-entrant jet (i.e., ejected material) is a necessary condition for the formation of waves in high speed impact phenomena. However, these investigations did not provide a quantitative framework for relating welding parameters to wave formation. 

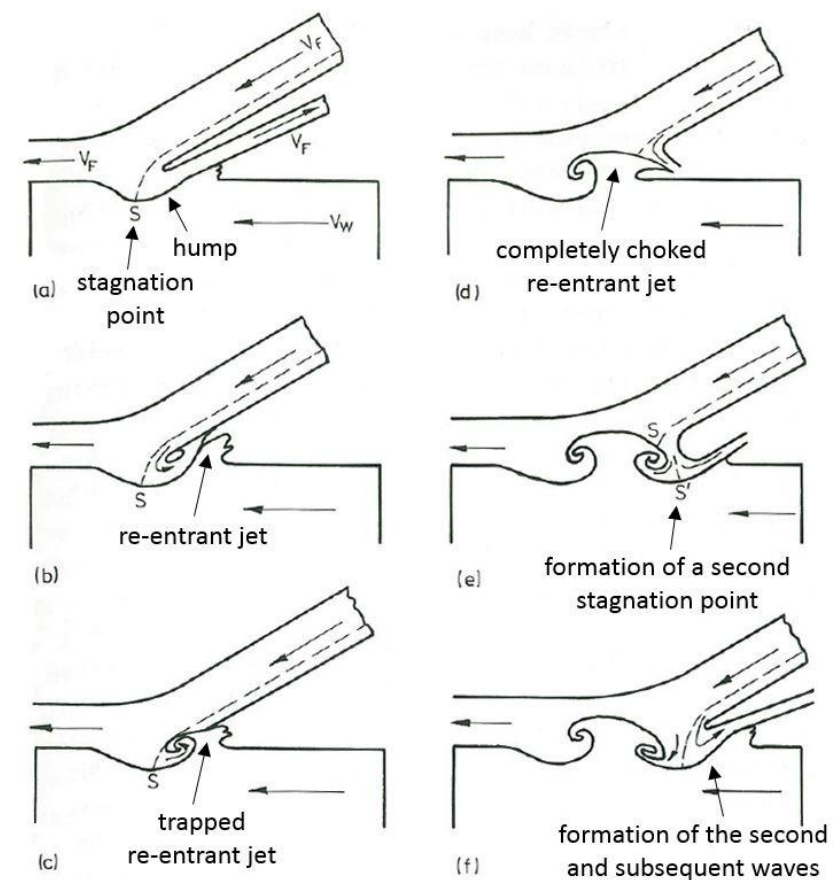

Fig. 3. Jet indentation mechanism for wave formation according to Bahrani et al. [12]

According to the second category (i.e., the stress wave mechanism), the interfacial waves are the result of successive interference from rarefaction waves in both plates, as first proposed by El-Sobki et al. [13] based on a fluid analogy. In this mechanism, the waves are generated ahead of the collision point. Note that these first two proposed wave formation mechanisms (i.e., the jet indentation and stress wave mechanisms) are better understood as conceptual frameworks rather than quantitative theories. Researchers later attempted to describe the formation of interfacial waves quantitatively, so that the conditions required for wave formation and the factors controlling the scale of the waves could be predicted.

Cowan et al. [14] were probably the first to recognize the similarities between the interfacial waves in EXW and the fluid flow around an obstacle, in which a regular pattern of eddies forms. Subsequently, this idea was extensively studied by Kowalick et al. [18], Reid [10] and Carton [19]. These authors posited that the waves are formed due to a vortex shedding mechanism (i.e., the third category) similar to that responsible for the formation of the von Kármán vortex street associated with the flow of a viscous fluid around an obstacle (see Fig. 4a). They speculated that in the neighborhood of the collision point, due to the extreme pressure, the flow can be considered as essentially Newtonian, with interfacial waves occurring 
above a critical value of a suitably defined Reynold number. In their studies, the dimension of the "obstacle" in the flow was presumed to scale with the jet thickness.

a)

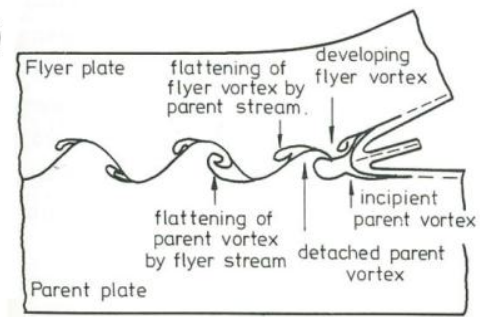

b)

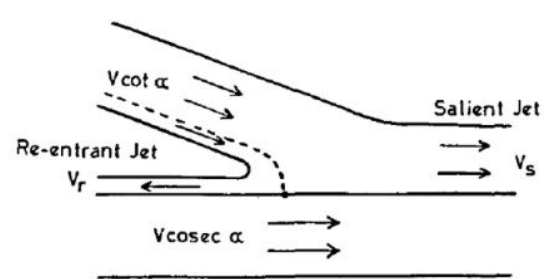

Fig. 4. a) Periodic wave formation by vortex shedding [18], b) incompressible jet formation referred to steady coordinates [15]

Yet another mechanism that has been proposed is based on a shear instability of the high strain-rate metal flow near the interface (i.e., the fourth category). Specifically, KelvinHelmholtz $(\mathrm{K}-\mathrm{H})$ instability, in which a tangential velocity difference at the interface induced by the presumed relative shearing motion of the flier and base plates provides an energy source for instability in the plastic deformation zone, has been implicated. For example, Hunt [15] and Wilson et al. [16] proposed that under extremely high pressure, the interface between the reentrant jet and base plate behaves as a fluid interface exhibiting a K-H instability (see Fig. 4b). Hunt also was the first to apply a quantitative hydrodynamic stability analysis in the study of EXW.

Robinson [17] proposed a complementary mechanism for wave formation based on a K-H instability. He showed that there exists a critical strain rate at which the materials begin to act as an inviscid fluid: above this critical strain rate, dislocations are unable either to move or to be generated. He suggested that wave formation depends on the critical strain rate of the particular metal, the impact velocity, the impact angle between the plates, and the thickness of the impacting plates. In the flow instability mechanism described by Robinson [17], the waves are created behind the collision point as a result of a velocity discontinuity across the interface between the base plate and the salient jet. This is contrary to the flow instability mechanism proposed by Hunt [15], in which the waves would be created ahead of the collision point (see Fig. 4b).

Further support for the proposition that wave formation results from a shear instability is provided by the comprehensive experimental and computational studies of EXW by Mousavi 
et al. [20], who argued that wave formation results from transverse variations in the velocity distribution near the collision point. Partly motivated by this work, including the notion that the instability could be introduced at a specific point, i.e., the collision point, Nassiri et al. [21] used spatial stability analysis to investigate the emergence of wavy morphology.

The interfacial grain deformation, namely, the highly elongated grains at the weld interface [1], also suggests that the wave formation mechanism could be associated with a flow process. Among these various proposed mechanisms, some researchers considered localized melting and rapid solidification to be responsible for joining and, hence, wave formation [22] , while others attributed the wave to high interfacial strain-rate deformation [23].

In this regard, it is important to note that, with the exception of the analysis by Nassiri et al. [21], the previous investigations of flow-instability mechanisms implicitly assumed melting by restricting attention to either inviscid or Newtonian-viscous fluid constitutive behavior within the shear zone. In reality, however, a good weld occurs when there is sufficient energy for plastic deformation but not for melting and, indeed, according to the previous studies, no heat affected zones have been reported [1]. If melting does not occur, a strictly fluid approach is inappropriate. Moreover, finite-element simulations of HVIW [1] reveal that the plastic flow profile near the interface is jet-like (symmetric) in contrast to the shear-layer-like (antisymmetric) profiles analyzed by previous investigators [15-17].

Here, a temporal linear stability analysis is performed to explore whether the wavy morphology observed in HVIW could be the signature of a shear-driven, high strain-rate instability of a perfectly plastic material. This analysis assumes that the disturbance amplifies in time but not in space. Crucially, neither melting nor relative shearing motion of the plates is presumed to occur. The notion underlying this model is that the extreme pressure at the interface, which is realized for a few tens of microseconds, causes a high strain rate process in which the yield stress of the material is exceeded for a short interval of time. During that time, a sharp variation in tangential velocity associated with a jet-like plastic flow in a narrow shear zone where the flier and base plates meet can drive instability and thereby account for the creation of the observed wavy morphology. 


\section{Model formulation}

In this section, a simplified mathematical model is formulated that is amenable to quantitative stability analysis yet retains those physical elements deemed to be essential to the instability mechanism proposed here. Specifically, the model incorporates a jet-like (symmetric) plastic flow near the interface and a tensorial constitutive model that, by construction, enforces perfectly-plastic material behavior and so does not imply melting. Although a thermo-mechanical material model that incorporates strain hardening, temperature, and strain rate-effects, e.g., the Johnson-Cook constitutive model, arguably would be more appropriate, for stability analysis the stress/strain (-rate) relationship generally is required in tensorial form (unlike the Johnson-Cook formula). More importantly, the primary aim of this investigation is not to replicate with quantitative realism every aspect of any given impact welding process, but rather to identify and understand the basic physical mechanisms that may control patterning in variety of similar impact welding scenarios. Consequently, in the present investigation, an abstracted mathematical model is formulated that retains a minimal set of geometric, material and physical elements; this model is then quantitatively analyzed (using linear stability analysis) to discern whether these elements are capable of generating wavy morphologies broadly compatible with those observed in welding processes. In this manner, hypothesized mechanisms can be understood and excluded or retained, and in this approach, complexity is added only as necessary.

\subsection{Constitutive model}

A simplification mode in this study is that the material behavior is perfectly plastic, thereby retaining strain-rate but not temperature nor strain-hardening effects. The following additional assumption are made: (i) Following impact, the two materials (i.e., plates) are treated as a single material with uniform material properties; (ii) The material behavior is isotropic; (iii) The stress and strain deviatorics are proportional; (iv) The flow is incompressible (since plasticity is essentially an incompressible phenomenon); and (v) The flow is two-dimensional and planar. As shown in Fig. 5, the coordinate system is defined such that the $x$-axis is aligned with the interface and weld direction, while the $z$-axis is oriented normal to the interface. 

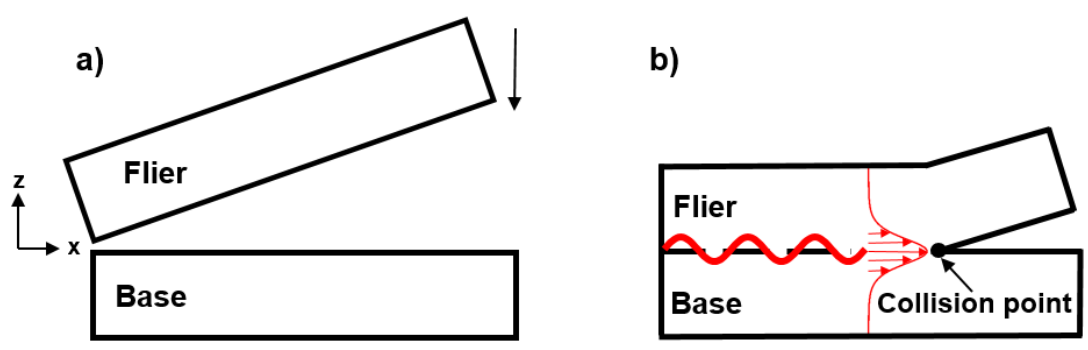

Fig. 5. Schematic of impact between two plates a) before impact, b) after impact

The flow is governed by the Cauchy equation

$$
\tilde{\rho} \frac{D \tilde{v}_{i}}{D \tilde{t}}=\frac{\partial}{\partial \tilde{x}_{j}}\left(\tilde{\sigma}_{i j}\right)+\tilde{f}_{i}
$$

and by the continuity condition for an incompressible material

$$
\frac{\partial \tilde{v}_{i}}{\partial \tilde{x}_{i}}=0
$$

In Eqs. (1) and (2), Cartesian indicial notation has been used (with $i, j=1,2$ and $1 \Leftrightarrow x, 2 \Leftrightarrow$ $z$ ), and tildes indicate dimensional quantities. Here, $\tilde{v}_{i}$ are the components of the velocity vector, $\tilde{\rho}$ is the material density, $\tilde{f}_{i}$ are the components of any body forces required to maintain the "base" plastic flow, and $\frac{D}{D \tilde{t}}$ is the material derivative. The stress tensor, $\tilde{\sigma}_{i j}$, in Eq. (1) is defined by [24]:

$$
\tilde{\sigma}_{i j}=-\tilde{p} \delta_{i j}+\frac{2 \tilde{\tau}_{s}}{\widetilde{H}} \dot{\tilde{\varepsilon}}_{i j}
$$

where $\tilde{p}$ is the scalar pressure field, $\delta_{i j}$ is the Kronecker delta, and $\tilde{\tau}_{s}$ is the scalar yield stress of the material. $\widetilde{H}$ is the intensity of the shear strain rate,

$$
\widetilde{H}=\sqrt{2 \dot{\tilde{\varepsilon}}_{i j} \dot{\tilde{\varepsilon}}_{i j}}
$$

where the strain-rate tensor

$$
\dot{\tilde{\varepsilon}}_{i j}=\frac{1}{2}\left(\frac{\partial \tilde{v}_{i}}{\partial \tilde{x}_{j}}+\frac{\partial \tilde{v}_{j}}{\partial \tilde{x}_{i}}\right)
$$


As is clear from Eq. (3), the stress components, $\tilde{\sigma}_{i j}$, include stress terms related to plastic deformation. Specifically, this constitutive relation satisfies $\left(\tilde{\sigma}_{i j}+\tilde{p} \delta_{i j}\right)\left(\tilde{\sigma}_{i j}+\tilde{p} \delta_{i j}\right)=2 \tilde{\tau}_{s}{ }^{2}$, so the material remains on the yield surface independently of the precise nature of the deformation, as required for a perfectly-plastic material. As a representative metal, Al6061-T6 is considered in this study. This material is a popular aluminum choice for many applications in manufacturing owing to its ability to be easily machined and its excellent weldability. The relevant physical properties of Al6061-T6 are: density $\tilde{\rho}=2700 \frac{\mathrm{kg}}{\mathrm{m}^{3}}$ and shear yield stress $\tilde{\tau}_{s}=34 \mathrm{MPa}$. Note that the value of the shear yield stress is based on the average temperature at the interface from finite-element simulations $\left(\sim 400^{\circ} \mathrm{C}\right)$ and on the high strain rates (approximately $10^{6} \mathrm{~s}^{-1}$ ) in VFAW processes [1].

\subsection{Basic state}

Classical linear stability theory begins with the decomposition of generic dependent variable $\widetilde{\boldsymbol{q}}$ into a steady state component $\widetilde{\boldsymbol{Q}}$, which is called the basic or base state, and an unsteady or perturbation field $\widetilde{\boldsymbol{q}}^{\prime}$,

$$
\widetilde{\boldsymbol{q}}(\boldsymbol{x}, t)=\widetilde{\boldsymbol{Q}}(\boldsymbol{x})+\widetilde{\boldsymbol{q}}^{\prime}(\boldsymbol{x}, t)
$$

where $\boldsymbol{x}$ is the spatial coordinate vector and $t$ is time. The aim of linear stability analysis is to determine whether a given basic state is susceptible to instability when perturbed by an arbitrary small disturbance. Substituting the decomposition (6) into the governing equations (i.e., the Cauchy Eq. (1) and continuity Eq. (2)) and neglecting terms that are quadratically small in the perturbation variables yields the linearized perturbation equations. Once the basic-state $\widetilde{\boldsymbol{Q}}$ has been specified, the linearized perturbation equations may be solved analytically or, more generally, numerically.

The choice of the basic state $\widetilde{\boldsymbol{Q}}$ around which the linearization is performed is not always straightforward. Here, the basic state is assumed to be a shear velocity profile, $\widetilde{\boldsymbol{U}}_{\boldsymbol{B}}(\boldsymbol{z})$; i.e., the base flow is assumed to be independent of time $\mathbf{t}$ and "streamwise" distance $\mathbf{x}$. Commonly occurring profiles in fluid dynamical contexts include shear layers, jets, and wakes (see Fig. 6). 


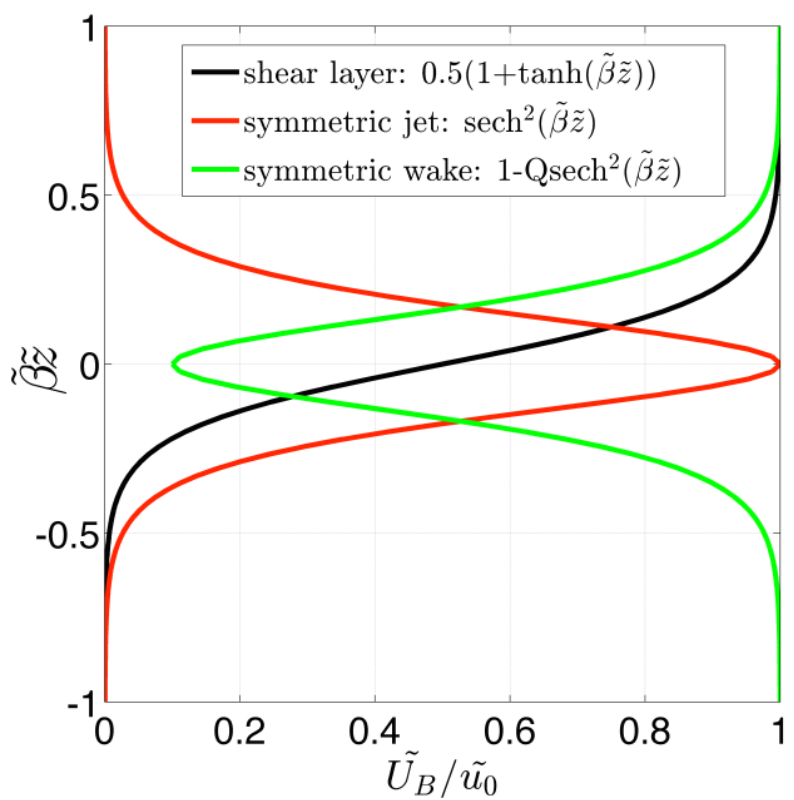

Fig. 6. Possible dimensionless base flow profiles across the interface between the two workpieces. The coordinate axes have been normalized using the velocity tangent to the interface $\left(\widetilde{\boldsymbol{u}}_{\mathbf{0}}\right)$ and the thickness of the region with significant shear $\left(\widetilde{\boldsymbol{\beta}}^{-\mathbf{1}}\right)$. Note that $\mathrm{Q}$ in the symmetric wake profile indicates the wake deficit, which is taken to be 0.9 in this figure.

To characterize the shape of the base velocity profile in HVIW, finite-element simulations were performed by Nassiri et al. [1]. The simulations show that the material near the collision point of both the flier and base workpieces moves with the same velocity (both magnitude and direction); see Fig. 7. Thus, a symmetric profile, i.e., a jet or wake depending on the reference frame chosen, is a reasonable abstraction. Again, apart from the investigation by Nassiri et al. [21], all previous theoretical studies of shear-driven instabilities in HVIW processes have treated anti-symmetric, that is, shear-layer base profiles. 


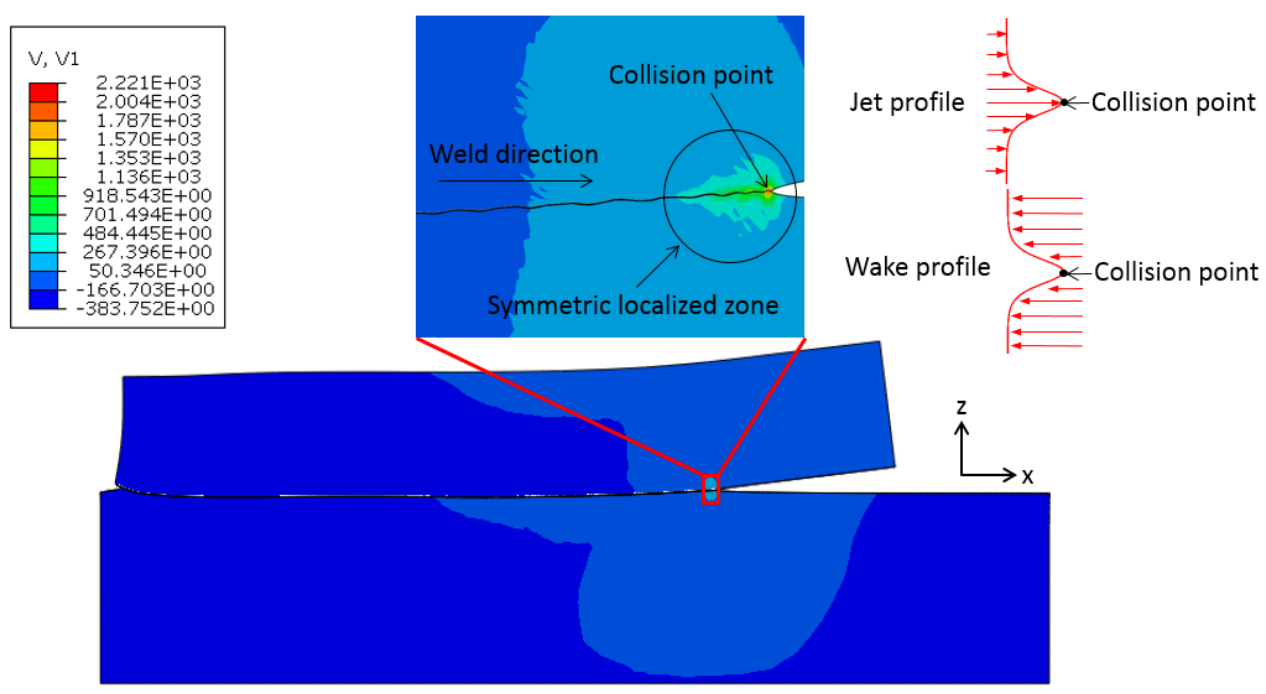

Fig. 7. Finite element simulation: symmetric $\boldsymbol{x}$-velocity profile along the interface [1]

Recall that the plastic stress terms in Eq. (3), $\tilde{s}_{i j} \equiv \tilde{\sigma}_{i j}+\tilde{p} \delta_{i j}$, ensure that the material always remains on the yield surface as it deforms. For a jet/wake base flow (or any profile evensymmetric about $\tilde{z}=0$ ), the basic-state plastic stress is always constant in magnitude but exhibits a jump in sign across $\tilde{z}=0$; see Fig. 8 .

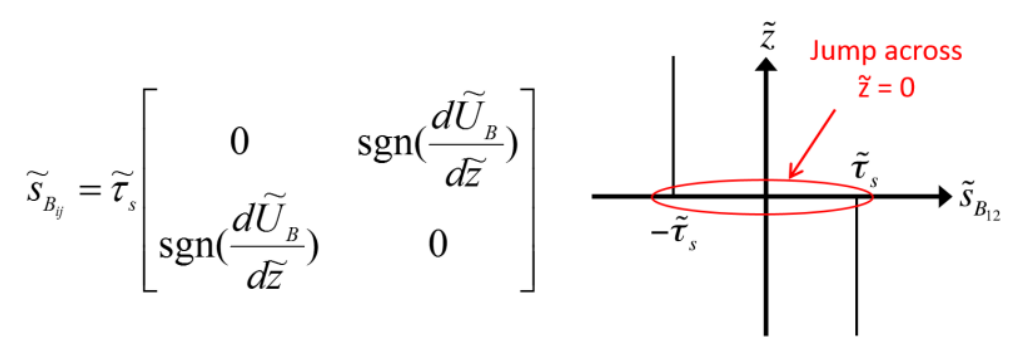

Fig. 8. Basic-state plastic stress in perfectly-plastic constitutive model for any shear profile that is evensymmetric about $\tilde{\mathbf{z}}=\mathbf{0}$ (see Eqs. (3), (13)-(15) and (17))

Thus, a basic state is sought for which:

$$
\begin{gathered}
\tilde{u}=\widetilde{U}_{B}(\tilde{z}) \\
\widetilde{w}=0 \\
\tilde{p}=\widetilde{P}_{B}(\tilde{x}, \tilde{z})
\end{gathered}
$$


Substituting Eqs. (7) and (8) into Eq. (2) confirms that the base (plastic) flow in the $x$-direction is a function only of the $\tilde{z}$ coordinate. To find the basic-state pressure distribution, the $\tilde{x}$ component of Eq. (1) (with $\widetilde{\tilde{x}_{\tilde{x}}}=0$ ) is used:

$$
\tilde{\rho}\left(\frac{\partial \widetilde{U}_{B}}{\partial \tilde{t}}+\widetilde{U}_{B} \frac{\partial \widetilde{U}_{B}}{\partial \tilde{x}}+\widetilde{w} \frac{\partial \widetilde{U}_{B}}{\partial \tilde{z}}\right)=\frac{\partial \tilde{\sigma}_{\tilde{x} \tilde{x}}}{\partial \tilde{x}}+\frac{\partial \tilde{\sigma}_{\tilde{x} \tilde{z}}}{\partial \tilde{z}}
$$

The left-hand side of Eq. (10) vanishes, implying

$$
\frac{\partial \tilde{\sigma}_{\tilde{x} \tilde{x}}}{\partial \tilde{x}}+\frac{\partial \tilde{\sigma}_{\tilde{x} \tilde{z}}}{\partial \tilde{z}}=0
$$

Substituting Eq. (3) into Eq. (11) yields

$$
\frac{\partial}{\partial \tilde{x}}\left(-\tilde{P}_{B}+\frac{2 \tilde{\tau}_{s}}{\widetilde{H}_{B}} \dot{\tilde{\varepsilon}}_{\tilde{x} \tilde{x}}\right)+\frac{\partial}{\partial \tilde{z}}\left(\frac{2 \tilde{\tau}_{s}}{\widetilde{H}_{B}} \dot{\tilde{\varepsilon}}_{\tilde{x} \tilde{z}}\right)=0
$$

where $\widetilde{H}_{B}$ is the basic-state shear strain-rate intensity.

From Eq. (5)

$$
\begin{gathered}
\dot{\tilde{\varepsilon}}_{\tilde{x} \tilde{x}}=\frac{\partial \tilde{u}}{\partial \tilde{x}}=\frac{\partial \widetilde{U}_{B}}{\partial \tilde{x}}=0 \\
\dot{\tilde{\varepsilon}}_{\tilde{x} \tilde{z}}=\dot{\tilde{\varepsilon}}_{\tilde{z} \tilde{x}}=\frac{1}{2}\left(\frac{\partial \tilde{u}}{\partial \tilde{z}}+\frac{\partial \widetilde{w}}{\partial \tilde{x}}\right)=\frac{1}{2} \frac{d \widetilde{U}_{B}}{d \tilde{z}} \\
\dot{\tilde{\varepsilon}}_{\tilde{z} \tilde{z}}=\frac{\partial \widetilde{w}}{\partial \tilde{z}}=0
\end{gathered}
$$

Using Eqs. (13) and (14) in Eq. (12) gives

$$
\frac{\partial}{\partial \tilde{x}}\left(-\widetilde{P}_{B}\right)+\frac{\partial}{\partial \tilde{z}}\left(\frac{\tilde{\tau}_{s}}{\widetilde{H}_{B}} \frac{d \widetilde{U}_{B}}{d \tilde{z}}\right)=0
$$

Using Eq. (4) and Eqs. (15)- (16), the basic-state shear strain-rate intensity is

$$
\widetilde{H}_{B}=\sqrt{2\left(\dot{\tilde{\varepsilon}}_{x x} \dot{\tilde{\varepsilon}}_{x x}+\dot{\tilde{\varepsilon}}_{x z} \dot{\tilde{\varepsilon}}_{x z}+\dot{\tilde{\varepsilon}}_{z x} \dot{\tilde{\varepsilon}}_{z x}+\dot{\tilde{\varepsilon}}_{z z} \dot{\tilde{\varepsilon}}_{z z}\right)}=\left|\frac{d \widetilde{U}_{B}}{d \tilde{z}}\right|
$$

Finally, from Eqs. (16)-(17), 


$$
\tilde{P}_{B}(\tilde{x}, \tilde{z})=\tilde{\tau}_{s} \tilde{x} \frac{\partial}{\partial \tilde{z}}\left[\operatorname{sgn}\left(\frac{d \widetilde{U}_{B}}{d \tilde{z}}\right)\right]
$$

Formally, the right-hand side of this expression is proportional to a delta distribution centered at the interface $\tilde{z}=0$; physically, the extreme pressure arising from impact of the plates and sustaining plastic deformation is thus idealized here as being strictly localized to the interface (much as an impulse in rigid-body dynamics is often modeled as a delta distribution in time). By similarly considering the $\tilde{z}$-component of the Cauchy equation for the basic-state, $\tilde{f}_{\tilde{z}}=\frac{\partial \tilde{P}_{B}}{\partial \tilde{z}}$, where $\tilde{f}_{\tilde{z}}=\tilde{f}_{\tilde{z}}(\tilde{x}, \tilde{z})$ is the sole non-zero body-force component.

\subsection{Linearized equations}

Having identified a suitable basic state, the analysis proceeds (as noted in Section 2.2) by decomposing all flow fields into basic-state and perturbation components,

$$
\begin{gathered}
\tilde{u}=\widetilde{U}_{B}(\tilde{z})+\tilde{u}^{\prime}(\tilde{x}, \tilde{z}, \tilde{t}) \\
\widetilde{w}=\widetilde{w}^{\prime}(\tilde{x}, \tilde{z}, \tilde{t}) \\
\tilde{p}=\tilde{P}_{B}(\tilde{x}, \tilde{z})+\tilde{p}^{\prime}(\tilde{x}, \tilde{z}, \tilde{t})
\end{gathered}
$$

where $\tilde{u}$ and $\widetilde{w}$ are dimensional streamwise and normal velocity components, $\tilde{p}$ is the dimensional pressure field, $\widetilde{U}_{B}$ and $\widetilde{P}_{B}$ are the base velocity and pressure, respectively, and $\tilde{u}^{\prime}$, $\widetilde{w}^{\prime}$, and $\tilde{p}^{\prime}$ represent the disturbance components of the flow variables. Next, these decompositions are substituted into the governing equations. For example, substituting Eqs. (19) and (20) into the continuity equation (Eq. (2)) yields

$$
\frac{\partial \tilde{u}^{\prime}}{\partial \tilde{x}}+\frac{\partial \widetilde{w}^{\prime}}{\partial \tilde{z}}=0
$$

The $\tilde{x}$-component of the Cauchy equation (Eq. (1)) requires

$$
\tilde{\rho}\left(\frac{\partial \tilde{u}}{\partial \tilde{t}}+\tilde{u} \frac{\partial \tilde{u}}{\partial \tilde{x}}+\widetilde{w} \frac{\partial \tilde{u}}{\partial \tilde{z}}\right)=\frac{\partial \tilde{\sigma}_{\tilde{x} \tilde{x}}}{\partial \tilde{x}}+\frac{\partial \tilde{\sigma}_{\tilde{x} \tilde{z}}}{\partial \tilde{z}}
$$

By substituting Eqs. (19) and (20) into Eq. (23) and neglecting products of perturbation variables (i.e., linearizing), the left-hand side of Eq. (23) simplifies to 


$$
\tilde{\rho}\left(\frac{\partial \tilde{u}^{\prime}}{\partial \tilde{t}}+\widetilde{U}_{B} \frac{\partial \tilde{u}^{\prime}}{\partial \tilde{x}}+\widetilde{w}^{\prime} \frac{d \widetilde{U}_{B}}{d \tilde{z}}\right)
$$

Substituting Eq. (3) into the right-hand side of Eq. (23) and using Eqs. (19)-(21) gives for the first term

$$
\frac{\partial \tilde{\sigma}_{\tilde{x} \tilde{x}}}{\partial \tilde{x}}=-\frac{\partial}{\partial \tilde{x}}\left(\widetilde{P}_{B}+\tilde{p}^{\prime}\right)+\frac{\partial}{\partial \tilde{x}}\left[\frac{2 \tilde{\tau}_{s}}{\widetilde{H}}\left(\frac{\partial}{\partial \tilde{x}}\left(\widetilde{U}_{B}+\tilde{u}^{\prime}\right)\right)\right]
$$

Upon linearizing and using Eq. (17),

$$
\frac{\partial \tilde{\sigma}_{\tilde{x} \tilde{x}}}{\partial \tilde{x}}=-\frac{\partial \tilde{p}^{\prime}}{\partial \tilde{x}}+\frac{2 \tilde{\tau}_{s}}{\left|\frac{d \widetilde{U}_{B}}{d \tilde{z}}\right|} \frac{\partial^{2} \tilde{u}^{\prime}}{\partial \tilde{x}^{2}}-\frac{\partial \tilde{P}_{B}}{\partial \tilde{x}}
$$

Employing Eq. (14),

$$
\frac{\partial \tilde{\sigma}_{\tilde{x} \tilde{z}}}{\partial \tilde{z}}=\frac{\partial}{\partial \tilde{z}}\left[\frac{2 \tilde{\tau}_{s}}{\widetilde{H}}\left(\frac{1}{2}\left(\frac{\partial\left(\widetilde{U}_{B}+\tilde{u}^{\prime}\right)}{\partial \tilde{z}}+\frac{\partial \widetilde{w}^{\prime}}{\partial \tilde{x}}\right)\right)\right]
$$

Linearizing Eq. (27) gives

$$
\begin{aligned}
\frac{\partial \tilde{\sigma}_{\tilde{x} \tilde{z}}}{\partial \tilde{z}} & =\tilde{\tau}_{s} \frac{\partial}{\partial \tilde{z}}\left(\frac{1}{\left|\frac{d \widetilde{U}_{B}}{d \tilde{z}}\right|}\left(\frac{\partial \tilde{u}^{\prime}}{\partial \tilde{z}}+\frac{\partial \widetilde{w}^{\prime}}{\partial \tilde{x}}\right)\right) \\
& +\tilde{\tau}_{s} \mathcal{L}\left(\frac{\partial}{\partial \tilde{z}}\left(\frac{1}{\widetilde{H}} \frac{d \widetilde{U}_{B}}{d \tilde{z}}\right)\right)
\end{aligned}
$$

where $\mathcal{L}$ in Eq. (28) denotes a linearization operator. Using Eq. (4) to expand the intensity of the shear strain rate,

$$
\frac{1}{\widetilde{H}} \equiv \frac{1}{\sqrt{2\left(\dot{\tilde{\varepsilon}}_{\tilde{x} \tilde{x}}^{2}+\dot{\tilde{\varepsilon}}_{\tilde{x} \tilde{z}}^{2}+\dot{\tilde{\varepsilon}}_{\tilde{z} \tilde{x}}^{2}+\dot{\tilde{\varepsilon}}_{\tilde{z} \tilde{z}}^{2}\right)}}
$$

where

$$
\dot{\tilde{\varepsilon}}_{\tilde{x} \tilde{x}}=\frac{\partial \tilde{u}^{\prime}}{\partial \tilde{x}}
$$




$$
\begin{gathered}
\dot{\tilde{\varepsilon}}_{\tilde{z} \tilde{z}}=\frac{\partial \widetilde{w}^{\prime}}{\partial \tilde{z}} \\
\dot{\tilde{\varepsilon}}_{\tilde{x} \tilde{z}}=\dot{\tilde{\varepsilon}}_{\tilde{z} \tilde{x}}=\frac{1}{2}\left(\frac{\partial \tilde{u}^{\prime}}{\partial \tilde{z}}+\frac{\partial \widetilde{w}^{\prime}}{\partial \tilde{x}}\right)+\frac{1}{2} \frac{d \widetilde{U}_{B}}{d \tilde{z}}
\end{gathered}
$$

Expanding Eq. (29) in a Taylor series about the basic state yields

$$
\mathcal{L}\left(\frac{1}{\widetilde{H}}\right) \equiv \frac{1}{\left|\frac{d \widetilde{U}_{B}}{d \tilde{z}}\right|}\left[1-\frac{1}{\frac{d \widetilde{U}_{B}}{d \tilde{z}}}\left(\frac{\partial \tilde{u}^{\prime}}{\partial \tilde{z}}+\frac{\partial \widetilde{w}^{\prime}}{\partial \tilde{x}}\right)\right]
$$

Substituting Eq. (33) into Eq. (28) then gives

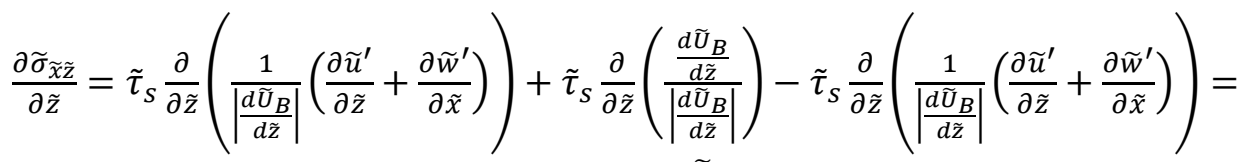

$$
\begin{aligned}
& \tilde{\tau}_{s} \frac{\partial}{\partial \tilde{z}}\left[\operatorname{sgn}\left(\frac{d \widetilde{U}_{B}}{d \tilde{z}}\right)\right]
\end{aligned}
$$

Finally, after canceling the basic-state terms, the linearized $\tilde{x}$-momentum equation is,

$$
\tilde{\rho}\left(\frac{\partial \tilde{u}^{\prime}}{\partial \tilde{t}}+\widetilde{U}_{B} \frac{\partial \tilde{u}^{\prime}}{\partial \tilde{x}}+\widetilde{w}^{\prime} \frac{d \widetilde{U}_{B}}{d \tilde{z}}\right)=-\frac{\partial \tilde{p}^{\prime}}{\partial \tilde{x}}+\frac{2 \tilde{\tau}_{s}}{\left|\frac{d \widetilde{U}_{B}}{d \tilde{z}}\right|} \frac{\partial^{2} \tilde{u}^{\prime}}{\partial \tilde{x}^{2}}
$$

The $\tilde{z}$-component of Eq. (1) is

$$
\tilde{\rho}\left(\frac{\partial \widetilde{w}}{\partial \tilde{t}}+\tilde{u} \frac{\partial \widetilde{w}}{\partial \tilde{x}}+\widetilde{w} \frac{\partial \widetilde{w}}{\partial \tilde{z}}\right)=\frac{\partial \tilde{\sigma}_{\tilde{z} \tilde{x}}}{\partial \tilde{x}}+\frac{\partial \tilde{\sigma}_{\tilde{z} \tilde{z}}}{\partial \tilde{z}}+\widetilde{f}_{\tilde{z}}
$$

Following a similar procedure, Eq. (36) can be linearized:

$$
\tilde{\rho}\left(\frac{\partial \widetilde{w}^{\prime}}{\partial \tilde{t}}+\widetilde{U}_{B} \frac{\partial \widetilde{w}^{\prime}}{\partial \tilde{x}}\right)=-\frac{\partial \tilde{p}^{\prime}}{\partial \tilde{z}}+2 \tilde{\tau}_{s} \frac{\partial}{\partial \tilde{z}}\left(\frac{1}{\left|\frac{d \widetilde{U}_{B}}{d \tilde{z}}\right|}\left(\frac{\partial \widetilde{w}^{\prime}}{\partial \tilde{z}}\right)\right)
$$

For ease of reference, the linearized system of equations in primitive variables is collected below.

Continuity:

$$
\frac{\partial \tilde{u}^{\prime}}{\partial \tilde{x}}+\frac{\partial \widetilde{w}^{\prime}}{\partial \tilde{z}}=0
$$


$\tilde{x}$-momentum: $\quad \tilde{\rho}\left(\frac{\partial \tilde{u}^{\prime}}{\partial \tilde{t}}+\widetilde{U}_{B} \frac{\partial \tilde{u}^{\prime}}{\partial \tilde{x}}+\widetilde{w}^{\prime} \frac{d \widetilde{U}_{B}}{d \tilde{z}}\right)=-\frac{\partial \tilde{p}^{\prime}}{\partial \tilde{x}}+\frac{2 \tilde{\tau}_{S}}{\left|\frac{d \widetilde{U}_{B}}{d \tilde{z}}\right|} \frac{\partial^{2} \tilde{u}^{\prime}}{\partial \tilde{x}^{2}}$

$\tilde{z}$-momentum: $\quad \tilde{\rho}\left(\frac{\partial \widetilde{w}^{\prime}}{\partial \tilde{t}}+\widetilde{U}_{B} \frac{\partial \widetilde{w}^{\prime}}{\partial \tilde{x}}\right)=-\frac{\partial \widetilde{p}^{\prime}}{\partial \tilde{z}}+2 \tilde{\tau}_{s} \frac{\partial}{\partial \tilde{z}}\left(\frac{1}{\left|\frac{d \widetilde{U}_{B} \mid}{d \tilde{z}}\right|}\left(\frac{\partial \widetilde{w}^{\prime}}{\partial \tilde{z}}\right)\right)$

It is convenient to work in dimensionless variables by selecting basic length and velocity scales of the flow. This is achieved for HVIW by defining the thickness of the region with significant shear, $\tilde{\beta}^{-1}$, as a relevant length scale and the characteristic speed of the base (plastic) flow tangent to the interface, $\tilde{u}_{0}$, as the velocity scale. The pressure can be nondimensionalized by $\tilde{\rho} \tilde{u}_{0}{ }^{2}$ and time by $\left(\tilde{u}_{0} \tilde{\beta}\right)^{-1}$. Table 1 summarizes this procedure; henceforth dimensionless variables are denoted without tildes. By substituting the scalings defined in Table 1 into Eqs. (38)-(40), the dimensionless, linearized system of equations is obtained.

Table 1: Non-dimensional variables

\begin{tabular}{|c|c|c|}
\hline Length & $x=\tilde{x} \tilde{\beta}$ & $z=\tilde{z} \tilde{\beta}$ \\
\hline Velocity & $u=\frac{\tilde{u}^{\prime}}{\tilde{u}_{0}}$ & $w=\frac{\widetilde{w}^{\prime}}{\tilde{u}_{0}}$ \\
\hline Time & \multicolumn{2}{|c|}{$t=\tilde{t} \tilde{u}_{0} \tilde{\beta}$} \\
\hline Pressure & \multicolumn{2}{|c|}{$p=\frac{\tilde{p}^{\prime}}{\tilde{\rho} \tilde{u}_{0}^{2}}$} \\
\hline
\end{tabular}

Continuity: $\quad \frac{\partial u}{\partial x}+\frac{\partial w}{\partial z}=0$

$x$-momentum:

$$
\frac{\partial u}{\partial t}+U_{B} \frac{\partial u}{\partial x}+w \frac{d U_{B}}{d z}=-\frac{\partial p}{\partial x}+\frac{2 \tau_{s}}{\left|\frac{d U_{B}}{d z}\right|} \frac{\partial^{2} u}{\partial x^{2}}
$$

Z-momentum:

$$
\frac{\partial w}{\partial t}+U_{B} \frac{\partial w}{\partial x}=-\frac{\partial p}{\partial \mathrm{z}}+2 \tau_{s} \frac{\partial}{\partial \mathrm{z}}\left(\frac{1}{\left|\frac{d U_{B}}{d \mathrm{z}}\right|}\left(\frac{\partial w}{\partial \mathrm{z}}\right)\right)
$$


The non-dimensional yield stress, $\tau_{s} \equiv \frac{\tilde{\tau}_{s}}{\widetilde{\rho} \widetilde{u}_{0}^{2}}$, arising in Eqs. (42) and (43) is seen to be a primary control parameter for the linearized dynamics. Also noteworthy is the observation gleaned from these equations that the (perturbation) plastic stresses provide resistance only to normal deformations.

\section{Temporal stability analysis}

In temporal (eigenvalue) stability analysis, the long-time evolution of a modal disturbance, parameterized by a given real $x$-wavenumber $k$, is predicted. Instability of the flow is indicated by one or more temporal eigenvalues (usually denoted $\sigma$ ) having positive real part(s). The real part of the eigenvalue thus defines the linear growth rate of the disturbance, whereas the imaginary part defines the circular frequency (or phase speed). Although temporal stability analysis is strictly applicable only for time-independent base states and HVIW is a transient process, temporal stability analysis may still be applied by making a "frozen coefficient" (i.e., quasi-static) approximation. The formal validity of this approximation requires that the time scale for perturbation amplification be much less than the process time scale, a condition that can be checked a posteriori.

\subsection{Stream function/vorticity formulation}

The dimensionless system of linearized equations, Eqs. (41)-(43), is based on primitive variables. It is convenient to eliminate the pressure terms by changing variables to a stream function/vorticity formulation. For two-dimensional motion in the $x-z$ plane, the (negative of the) $y$-component of the vorticity, $\Omega$, (i.e., twice the local material rotation rate about a $y$-axis) is given by the (negative) curl of the velocity field:

$$
\Omega=\frac{\partial w}{\partial x}-\frac{\partial u}{\partial z}
$$

The stream function, $\psi$, is related to the $x$ and $z$ perturbation velocity components via

$$
u=-\frac{\partial \psi}{\partial z}, w=\frac{\partial \psi}{\partial x}
$$

Thus, lines of constant $\psi$ coincide with perturbation streamlines of the disturbed base flow. Substituting Eq. (45) into Eq. (44) provides a relationship between $\psi$ and $\Omega$, while subtraction of the $z$-derivative of Eq. (42) from the $x$-derivative of Eq. (43) yields an evolution equation for 
the (negative) $y$-vorticity component, $\Omega$. Thus, in stream function/vorticity form, the linearized equations governing the evolution of the perturbation fields can be expressed as

$$
\begin{gathered}
\nabla^{2} \psi=\Omega \\
\frac{\partial \Omega}{\partial t}+U_{B}(z) \frac{\partial \Omega}{\partial x}-\frac{d^{2} U_{B}}{d z^{2}} \frac{\partial \psi}{\partial x}=4 \frac{\partial}{\partial \mathrm{z}}\left(\frac{\tau_{s}}{\left|\frac{d U_{B}}{d \mathrm{z}}\right|} \frac{\partial}{\partial \mathrm{z}}\left(\frac{\partial^{2} \psi}{\partial x^{2}}\right)\right)
\end{gathered}
$$

where $\nabla^{2}$ indicates the Laplacian operator, which in two-dimensional Cartesian coordinates is given by $\nabla^{2}=\frac{\partial^{2}}{\partial x^{2}}+\frac{\partial^{2}}{\partial z^{2}}$.

Inspection of Eq. (47) indicates that the parameter combination $\frac{\tau_{S}}{\left|\frac{d U_{B}}{d z}\right|}$ is a dimensionless effective diffusion coefficient associated with plastic deformation. This term is a function of the base flow profile and hence of the $z$ coordinate.

\subsection{Normal mode analysis}

Eqs. (46) and (47) admit separable solutions. A single mode can be represented as

$$
\left[\begin{array}{l}
\Omega \\
\psi
\end{array}\right]=\left[\begin{array}{l}
\widehat{\Omega}(z) \\
\widehat{\psi}(z)
\end{array}\right] e^{i k x} e^{\sigma t}+C . C .
$$

where $\widehat{\Omega}(z)$ and $\widehat{\psi}(z)$ are vertical eigenfunctions, $k$ is the presumed real wavenumber, $\sigma=\sigma_{r}+$ $i \sigma_{i}$ defines the complex eigenvalue, and C.C. denotes complex conjugate. Subscripts $r$ and $i$ denote the real and imaginary parts of a complex quantity, respectively. Since Eqs. (46) and (47) comprise a linear system, the response of the base flow to an arbitrary initial disturbance can be determined by separately analyzing the response to these individual modes.

Substituting Eq. (48) into Eqs. (46) and (47) and denoting $z$-differentiation by $D \equiv \frac{d}{d z}$, the following eigenvalue problem is obtained:

$$
\left(D^{2}-k^{2}\right) \hat{\psi}-\widehat{\Omega}=0
$$




$$
\sigma \widehat{\Omega}=\left(-i k U_{B}\right) \hat{\Omega}+\left[i k D^{2} U_{B}-4 \tau_{s} k^{2} D\left(\frac{1}{\left|\frac{d U_{B}}{d \mathrm{z}}\right|}\right) D\right] \widehat{\psi}
$$

Eqs. (49) and (50) are solved numerically for the eigenvectors $\hat{\Omega}$ and $\hat{\psi}$ and associated eigenvalue $\sigma$. The sign of the real part of the eigenvalue, $\sigma_{r}$, determines whether exponential growth and hence instability is possible.

Although Eqs. (49) and (50) are linear, the latter equation has non-constant coefficients that are functions of the $z$ coordinate. In general, this renders a strictly analytical treatment impossible, so a numerical method, in which the $z$ coordinate is discretized, is required. Rather than using a low-order finite-difference or finite-element discretization, here a highly accurate and low-storage Chebyshev spectral collocation method, in which the unknown fields are expanded in Chebyshev series in the $z$ direction, is employed [25].

Following discretization in $z$ using this Chebyshev spectral collocation method, Eqs. (49) and (50) can be expressed as an algebraic generalized eigenvalue problem for $\sigma$ of the form

$$
\mathrm{A} V=\sigma \mathrm{B} V
$$

where

$$
\begin{gathered}
\mathrm{A}=\left[\begin{array}{cc}
{\left[-i k U_{B}\right]} & {\left[i k D_{N}{ }^{2} U_{B}-4 \tau_{S} k^{2} D_{N} \frac{1}{\left|\frac{d U_{B} \mid}{d z}\right|} D_{N}\right.} \\
{[-I]} & {\left[D_{N}{ }^{2}-k^{2}\right]}
\end{array}\right] \\
\mathrm{B}=\left[\begin{array}{ll}
{[I]} & {[0]} \\
{[0]} & {[0]}
\end{array}\right] \\
V=\left[\begin{array}{l}
\hat{\Omega} \\
\hat{\psi}
\end{array}\right]
\end{gathered}
$$

where $I$ is the identity matrix, and $D_{N}$ is the first-order Chebyshev differentiation matrix [25]. Note that the A and B matrices are actually composed of four submatrices, each of length $N+$ 1 , where $N$ is the resolution in the $z$-direction. This algebraic eigenvalue problem is solved numerically using the QZ algorithm. 


\subsection{Boundary conditions}

To complete the problem specification, Eqs. (46) and (47) must be supplemented by appropriate boundary conditions. For computational efficiency, the symmetry of the base flow is exploited so that only half the $z$-domain $0 \leq z \leq h$, where $h$ is half the dimensionless thickness of the welded material, is considered (see Fig. 9).

Along the upper boundary of the domain, i.e., along $z=h$, the condition $\psi=0$ is imposed to ensure that the normal (i.e., $z$ ) velocity component vanishes there. The appropriate constraint to be imposed along the symmetry plane, $z=0$, is more subtle. For symmetric baseflow profiles, including the jet profile considered in this study, $\frac{d U_{B}}{d z}=0$ along $z=0$; hence, the effective diffusion coefficient $\left(\frac{\tau_{s}}{\left|\frac{d U_{B}}{d z}\right|}\right)$ arising in Eq. (47) is unbounded there. Referring to the perturbation $x$-momentum equation (Eq. (42)), it seems reasonable physically that the effectively infinite resistance to normal deformations along $z=0$ causes the perturbation $x$ velocity component, $\equiv \frac{\partial \psi}{\partial z}$, to vanish along the symmetry plane. This constraint also appears plausible mathematically, since by the continuity Eq. (41), then $\frac{\partial w}{\partial z}$ also vanishes along $z=0$, potentially rendering the solution behavior for $w$ regular there; cf. the last term on the righthand side of Eq. (43).

Unfortunately, our numerical computations with $\psi=0$ along $z=h$ and $\frac{\partial \psi}{\partial z}=0$ along $z=0$ proved to be badly ill-conditioned, indicating the necessity of a more careful mathematical treatment. As detailed in the Appendix, a local analysis of the eigensystem given by Eqs. (49)-(50) confirms that $z=0$ is a regular singular point (a non-essential singularity). Thus, the local functional form of the solution $\hat{\psi}$ (and, thence, $\hat{\Omega}$ ) can be determined using the method of Frobenius. Specifically, the following asymptotic expansion for $\hat{\psi}$ is posited as $z \rightarrow$ $0^{+}:$

$$
\hat{\psi} \sim \sum_{n=0}^{\infty} \psi_{n} Z^{(n+r)}
$$

As shown in the Appendix, the indicial exponent $r=0,2$, corresponding to two linearlyindependent solution behaviors; i.e., although the two $r$ values differ by an integer, logarithmic dependence on $z$ is not found and the solution is, indeed, smooth near $z=0$. Nevertheless, to 
avoid numerical division by zero, the original boundary condition $\frac{\partial \psi}{\partial z}=0$ at $z=0$ is replaced by the two supplementary conditions (A.37) and (A.39) derived in the Appendix, which are imposed at $z=\delta$, where the parameter $\delta$ may be arbitrarily chosen as long as $0<\delta \ll 1$; see section 4.2. These effective boundary conditions are derived by exploiting the known form of the solution near $z=0$. Note that the strategy of deriving extrapolated, effective boundary conditions to avoid a boundary singularity in the numerical solution of differential equations is widely employed in a variety of contexts [26, 27].

Fig. 9 provides a schematic representation of the dimensional physical domain, the dimensionless physical domain, and the mapped (dimensionless) numerical domain, along with the location of the boundary condition(s) at (or near to) the symmetry plane $(z=0)$.

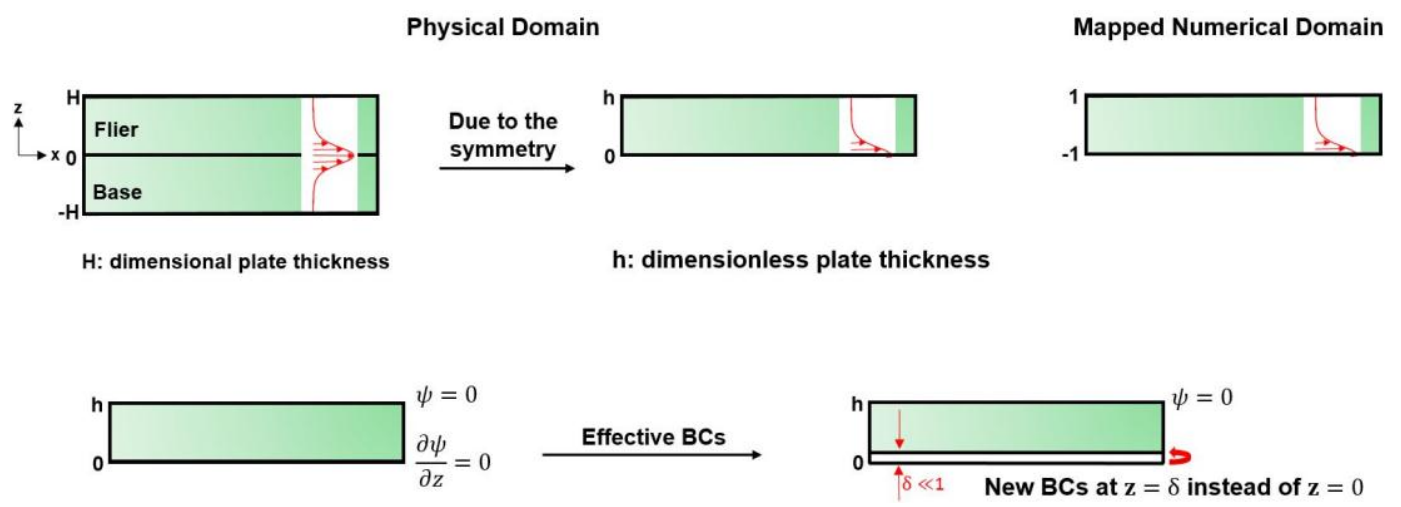

Fig. 9. Schematic of the dimensional physical domain, the dimensionless physical domain, and the mapped numerical domain with the location for the effective boundary conditions (BCs) indicated

\section{Results}

As noted in section 3, in temporal stability analysis the wavenumber $k$ is constrained to be a real number. Physically, this implies that only those wavelike disturbances that do not grow or decay in the streamwise direction will be considered; instead, the possibility of growth or decay of waves in time is examined. The dimensionless parameter $\tau_{s}$ arising in the governing equations is varied to investigate its effect on the stability of the model system. Different values of $\tau_{s}$ can be interpreted as corresponding to different values of the (plastic) jet centerline velocity (see Table 2 ). 
Table 2: Dimensionless shear yield stress values

\begin{tabular}{|c|c|c|c|}
\cline { 2 - 4 } \multicolumn{1}{c|}{} & $\widetilde{\boldsymbol{u}}_{\mathbf{0}} \approx \mathbf{1 0 0 0} \mathbf{m} / \mathbf{s}$ & $\widetilde{\boldsymbol{u}}_{\mathbf{0}} \approx \mathbf{1 0 0} \mathbf{m} / \mathbf{s}$ & $\widetilde{\boldsymbol{u}}_{\mathbf{0}} \approx \mathbf{1 0 ~} \mathbf{m} / \mathbf{s}$ \\
\hline $\boldsymbol{\tau}_{\boldsymbol{s}}=\frac{\tilde{\boldsymbol{\tau}}_{\boldsymbol{s}}}{\widetilde{\boldsymbol{\rho}}_{\tilde{\boldsymbol{u}}_{\mathbf{0}}{ }^{2}}}$ & 0.0126 & 1.26 & 126 \\
\hline
\end{tabular}

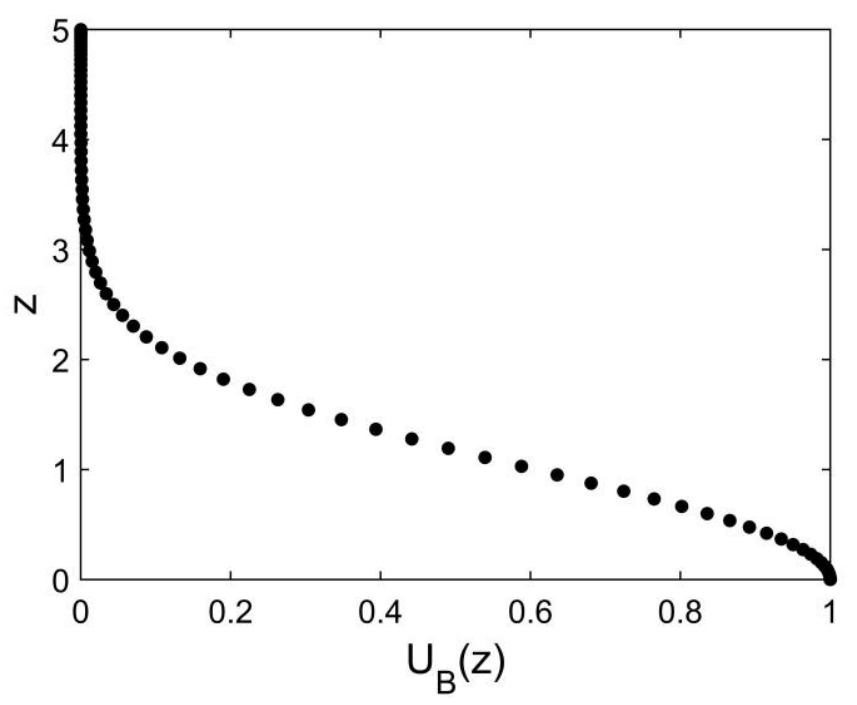

Fig. 10. Base velocity profile $(\boldsymbol{N}=\mathbf{8 0})$

Fig. 10 shows the symmetric jet base velocity profile (i.e., for half of the domain) that is used to obtain the results presented in this study. In Fig. 10, the number of Chebyshev grid points $N=80$. Note that the Gauss-Lobatto-Chebyshev grid points are distributed nonuniformly with a higher concentration of nodes close to the top and bottom of the domain. 


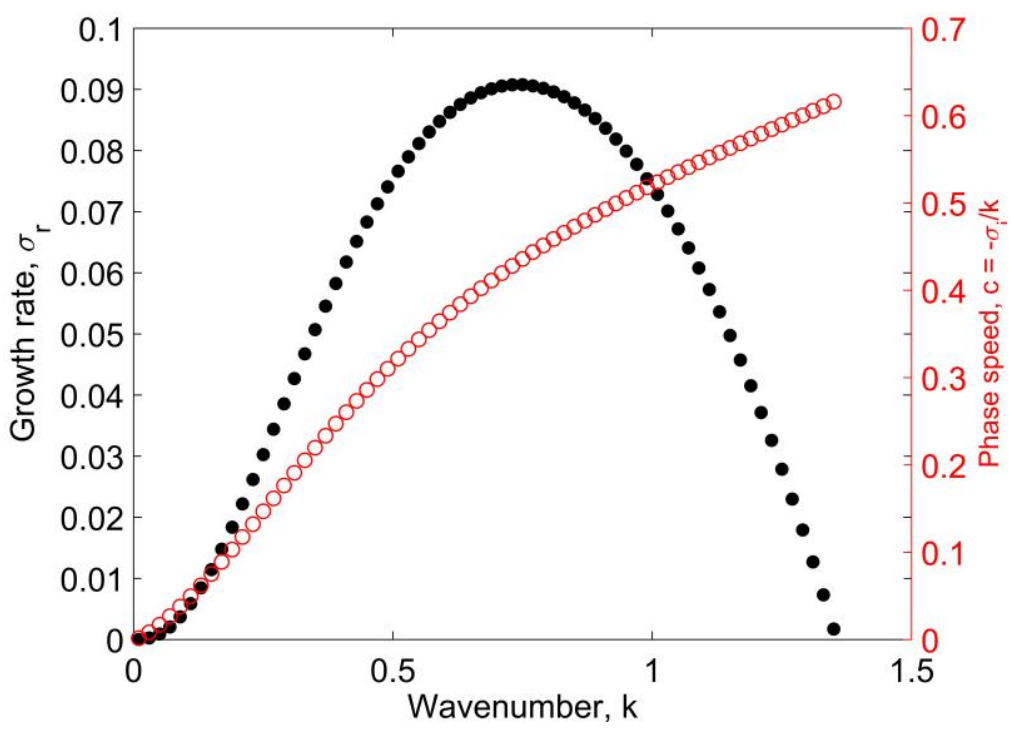

Fig. 11. Temporal growth rate (filled circle) $\boldsymbol{\sigma}_{\boldsymbol{r}}$ and phase speed $\boldsymbol{c}=-\frac{\boldsymbol{\sigma}_{\boldsymbol{i}}}{\boldsymbol{k}}$ as a function of wavenumber $\boldsymbol{k}$ $\left(N=80, \tau_{s}=0.0126\right)$

Fig. 11 shows the temporal growth rate $\sigma_{r}$ and the phase speed $c$ as a function of wavenumber $k$ for the case that $N=80$ and $\tau_{s}=0.0126$ (i.e., the case for which the jet centerline speed is on the order of $1000 \mathrm{~m} / \mathrm{s}$ ). Since $\sigma_{r}>0$ for $0<k<1.36$, instability is possible for these parameters. The fastest growing (i.e., the most "dangerous") mode occurs at $k=0.75$, for which $\sigma_{r}=0.09078$. The phase speed of this mode $c=-\frac{\sigma_{i}}{k}=0.4359$ indicates that the wave propagates in the $x$ (weld) direction as it is amplified at a speed that is roughly $44 \%$ of the jet centerline velocity.

Eigenvectors and two-dimensional eigenfunctions associated with the fastest growing mode (i.e., with $k=0.75$ and $\sigma_{r}=0.09078$ ) are depicted in Fig. 12, the latter showing the emergent wavy morphology. Figs. 12a and 12b show one-dimensional eigenvectors for the vorticity and stream function, respectively. Clearly, most of the support of these eigenfunctions occurs near the bottom of the domain where the strong shear exists, with the disturbances decaying as the top of the domain is approached. Figs. 12c and $12 \mathrm{~d}$ show two-dimensional eigenfunctions for the vorticity and stream function, respectively. In Fig. 12c, contours indicate the intensity of the local spinning motion of the material, with the signs indicating the direction of rotation. The strongly tilted contours in Fig. 12d reflect energy extraction by the 
disturbances via tilting into the shear (an "Orr" mechanism). Since the flow is periodic in the $x$ direction, the non-dimensional wavelength of the most amplified disturbance $\lambda=\frac{2 \pi}{k}=8.37$.

a)

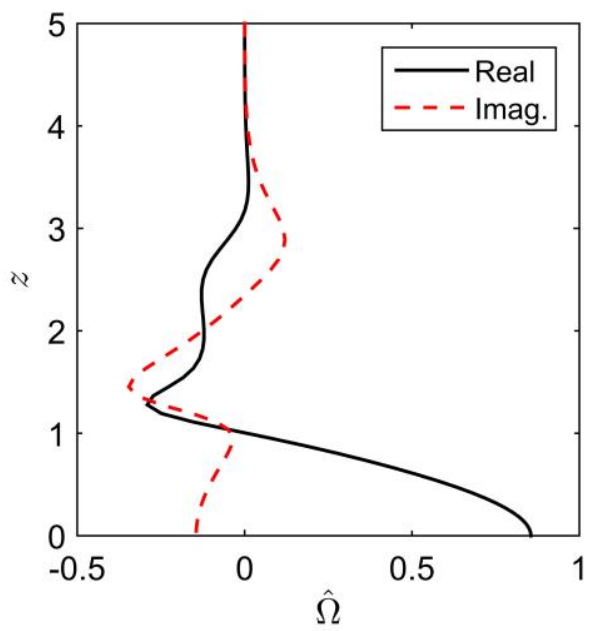

c)

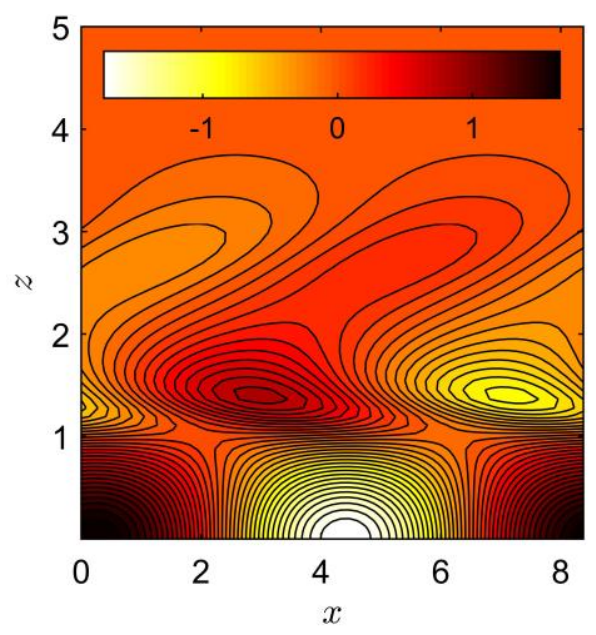

b)

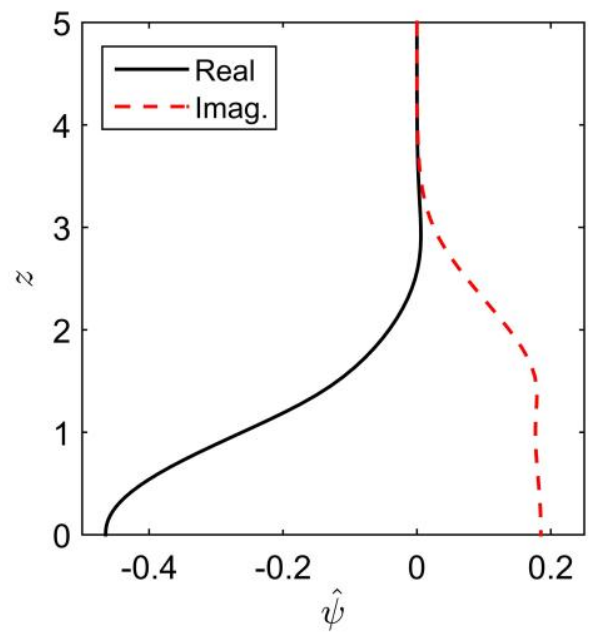

d)

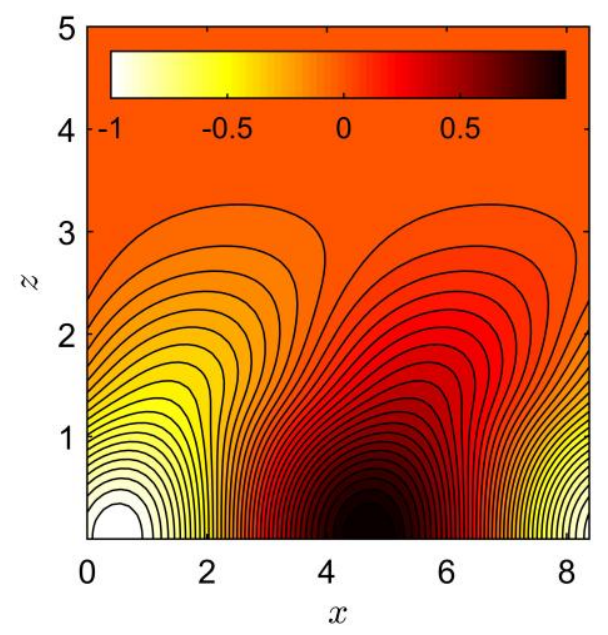

Fig. 12. Fastest-growing disturbance mode: one-dimensional eigenvectors a) vorticity, b) stream function and two-dimensional eigenfunctions c) vorticity, d) stream function $\left(\boldsymbol{N}=\mathbf{8 0}, \boldsymbol{\tau}_{\boldsymbol{s}}=\mathbf{0 . 0 1 2 6}\right)$

\subsection{Spatial resolution: Variation in $\mathbf{N}$}

The effect of the spatial resolution (i.e., the number of Chebyshev points, $\mathrm{N}$ ) on the predicted growth rate is shown in Fig. 13 for the case that the jet velocity is on the order of $1000 \mathrm{~m} / \mathrm{s}$ (i.e., for $\tau_{s}=0.0126$ ). 


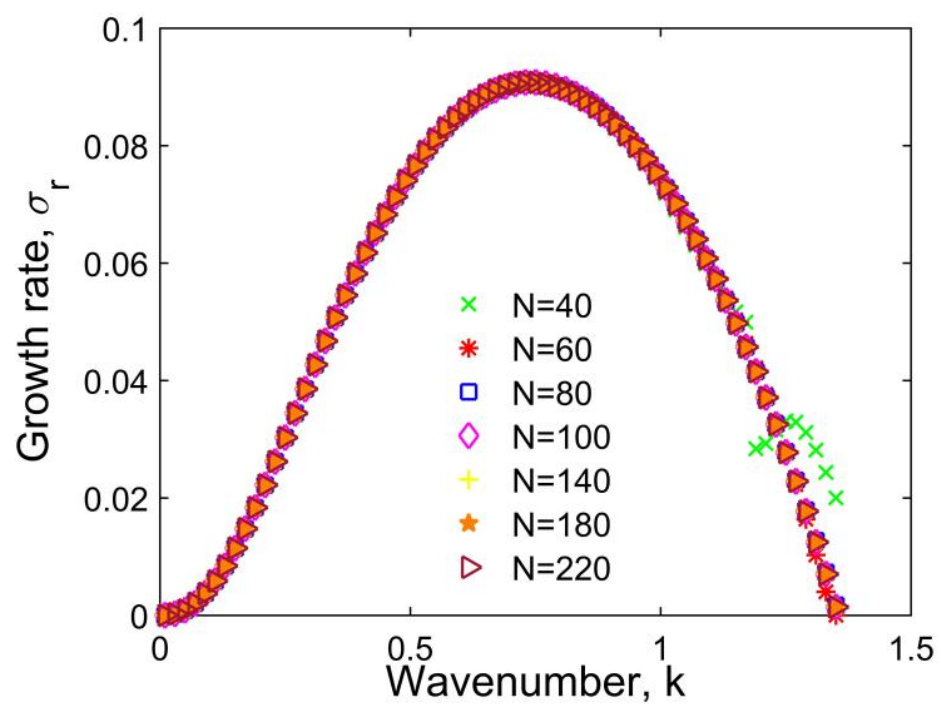

Fig. 13. Effect of the number of grid points $\mathrm{N}$ on the growth rate $\left(\boldsymbol{\tau}_{\boldsymbol{s}}=\mathbf{0 . 0 1 2 6}\right)$

This figure illustrates that once the number of grid points exceeds about 80 , there is little variation in the predicted disturbance growth rate. Note that this is not the case when the original symmetry boundary condition, $\frac{\partial \psi}{\partial z}=0$, is imposed; i.e., the results then show extreme sensitivity to the grid resolution. Thus, for the remaining calculations, the number of Chebyshev grid points is fixed at $\mathrm{N}=80$.

\subsection{Effective boundary conditions: Variations in $\delta$}

The effect of variations in the parameter $\delta$, the $z$-location at which the effective boundary conditions are imposed, on the predicted growth rate is shown in Fig. 14 for the case when the jet velocity is roughly $1000 \mathrm{~m} / \mathrm{s}$ (i.e., for $\tau_{s}=0.0126$ ). 

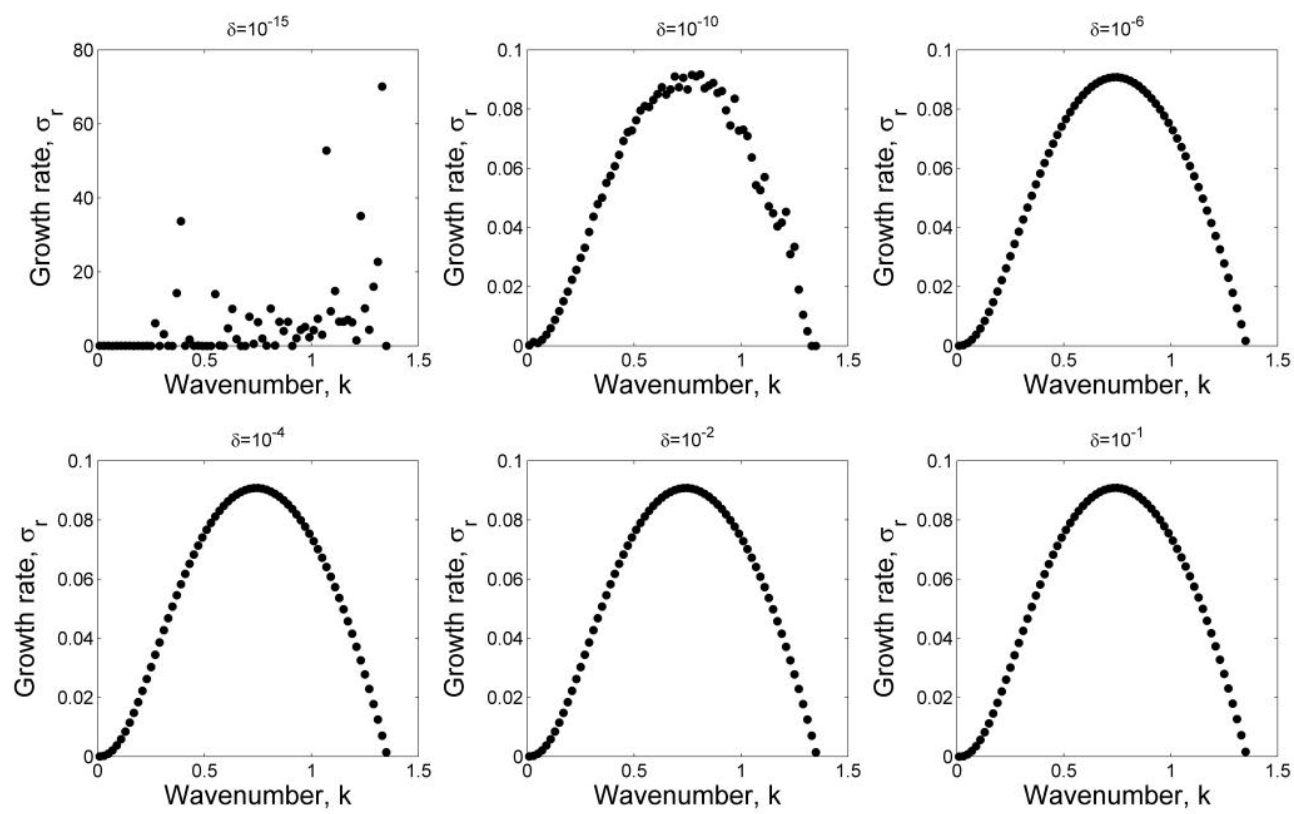

Fig. 14. Effect of $\delta$ on the growth rate $\left(\mathrm{N}=80\right.$ and $\left.\boldsymbol{\tau}_{\boldsymbol{s}}=\mathbf{0 . 0 1 2 6}\right)$

As expected, when $\delta$ is extremely small $\left(\delta<10^{-9}\right)$, the computational problem is illconditioned owing to near division by zero in the evaluation of the effective diffusion coefficient $\frac{\tau_{S}}{\left|\frac{d U_{B}}{d z}\right|}$ at $z=\delta$. Fig. 14 confirms, however, that there is a range of not too small nor too large $\delta$ values for which the eigenvalue distribution is smooth and converges, independently of the precise value chosen. This study along with that reported in Section 4.1 confirms that the stability results are robust when the effective boundary conditions, Eqs. (A.37) and (A.39), are employed. For the remaining calculations, $\delta$ is set equal to $10^{-6}$.

\subsection{Jet velocity: Variations in $\tau_{s}$}

The effect of varying $\tau_{s}$ (i.e., varying the centerline jet velocity for fixed material properties) on the growth rates and phase speeds of the fastest growing modes is shown in Fig. 15. Numerical simulations $[1,20]$ indicate that the jet velocity at the collision point should exceed $1000 \mathrm{~m} / \mathrm{s}$ for a successful weld. Experimentally, this can be investigated by creating a Weldability Window (WW). The WW incorporates key control parameters in the process such as collision velocity, impact velocity, and impact angle that result in a successful weld [28-31]. Clearly, by increasing the jet velocity the system is more prone to become unstable. Conversely, 
the trend evident in Fig. 15 (left) confirms that by decreasing the jet velocity sufficiently, the system can be stabilized. For example, the fastest growing mode for $\tau_{s}=1.26$ occurs at $k=$ 0.59 for which $\sigma_{r}=0.00062$, while for $\tau_{s}=126$, it occurs at $k=0.55$ with $\sigma_{r}=6.25 \times 10^{-5}$. Fig. 15 (right) compares the phase speed for different jet velocities. For $k<1$, the phase speed decreases as $\tau_{s}$ increases, but for $k>1$, the trend is reversed.
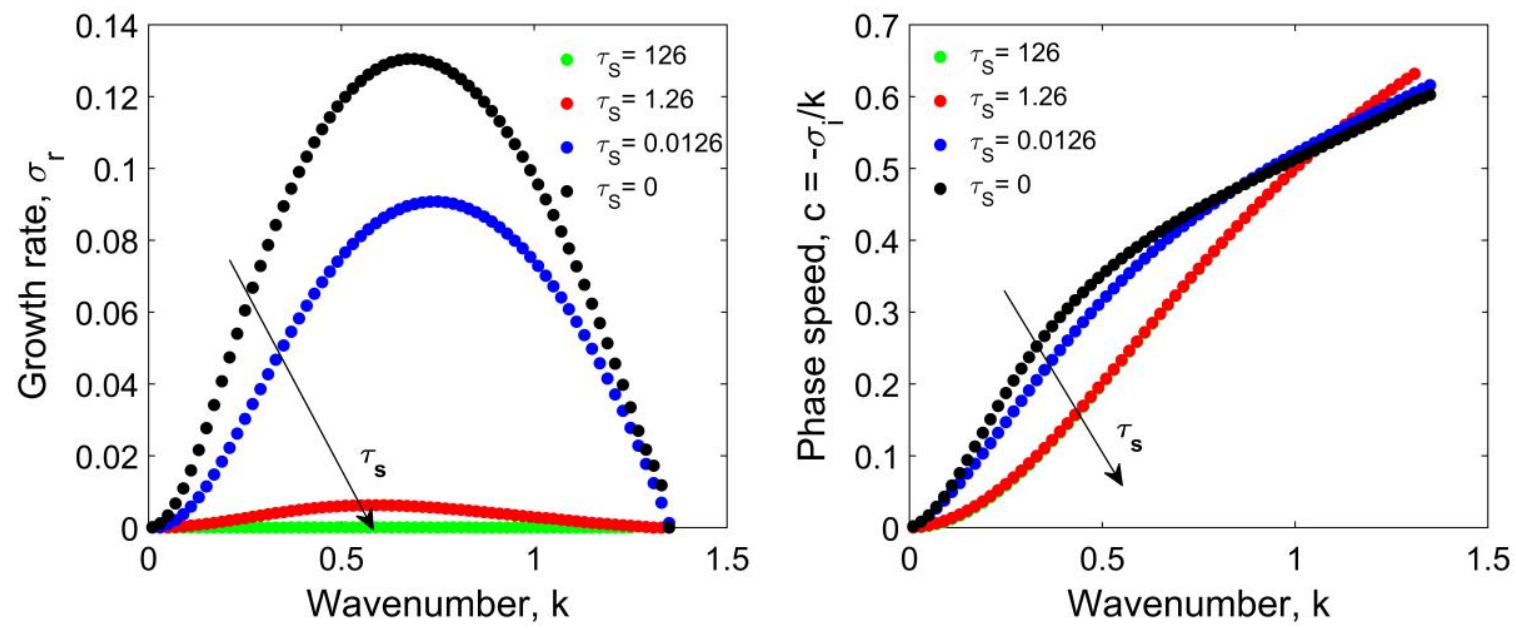

Fig. 15. Effect of jet velocity on growth rate (left) and phase speed (right) $(\boldsymbol{N}=\mathbf{8 0})$

When $\tau_{s}=0$, the classical stability problem for an inviscid fluid is recovered. The results obtained for these parameter values exactly match those for this classical problem, providing confidence in the formulation of the equations and the effective boundary conditions and in the programming of the numerical algorithm.

Figs. 16 and 17 show the one-dimensional eigenvectors and two-dimensional eigenfunctions for the fastest-growing disturbance modes for the cases with $\tau_{s}=1.26$ and $\tau_{s}=126$, respectively. Comparing with the eigenfunctions shown in Fig. 12 for the scenario with $\tau_{s}=0.0126$, it is seen that the vortices do not incline strongly into the shear, consistent with the reduced growth rates exhibited by these modes. 
a)

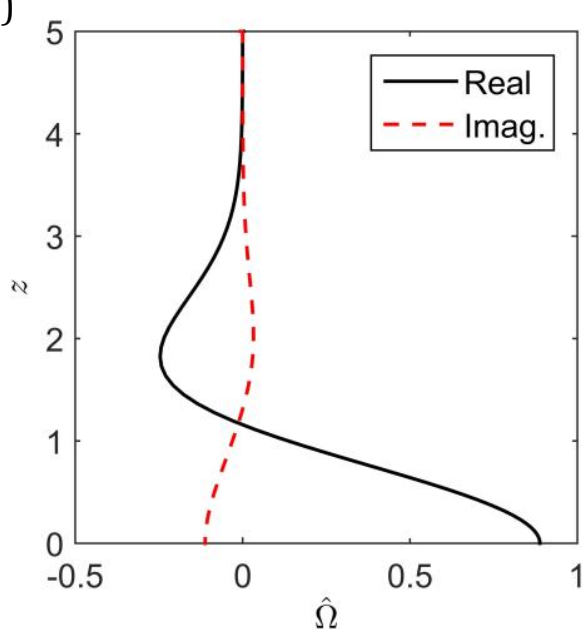

c)

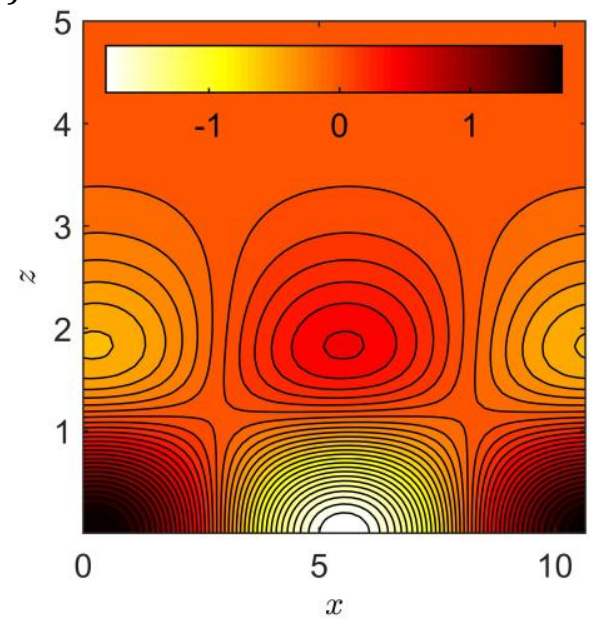

b)

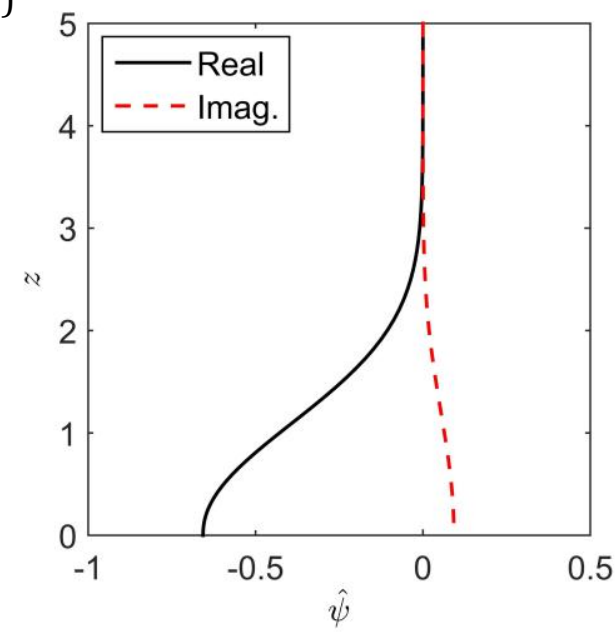

d)

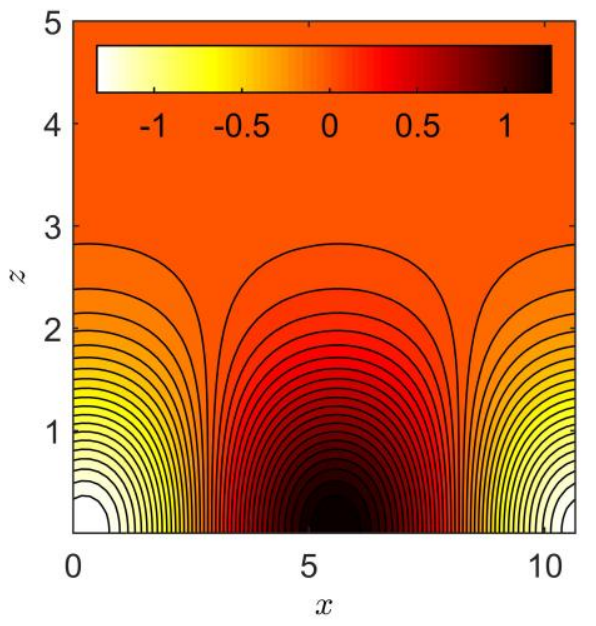

Fig. 16. Fastest-growing disturbance mode: one-dimensional eigenvectors a) vorticity, b) stream function and two-dimensional eigenfunctions c) vorticity, d) stream function $\left(\boldsymbol{N}=\mathbf{8 0}, \boldsymbol{\tau}_{\boldsymbol{s}}=\mathbf{1}\right.$. 26) 
a)

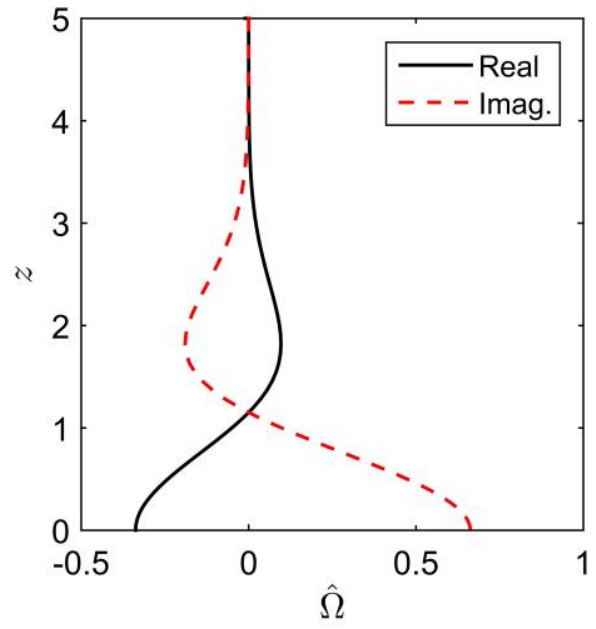

c)

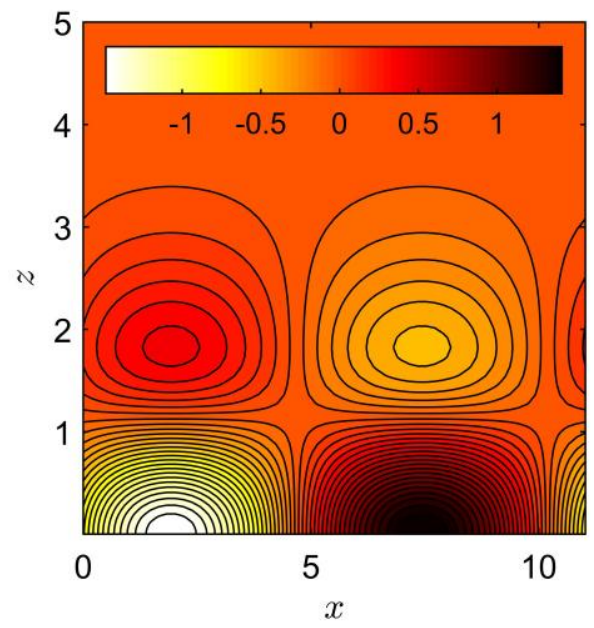

b)

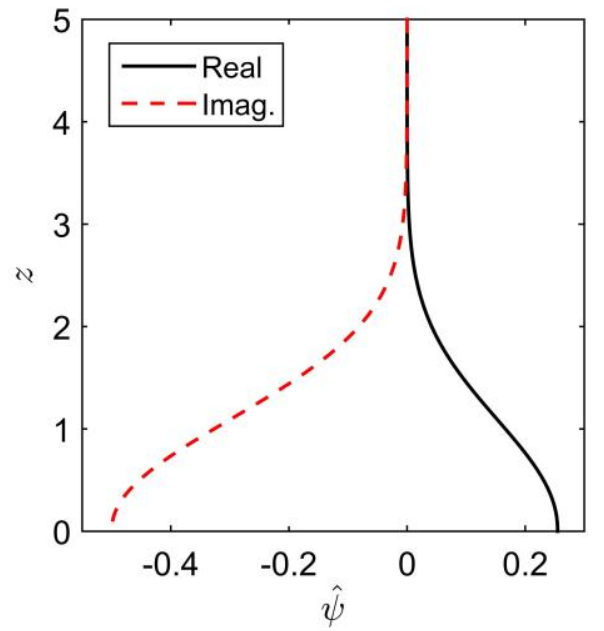

d)

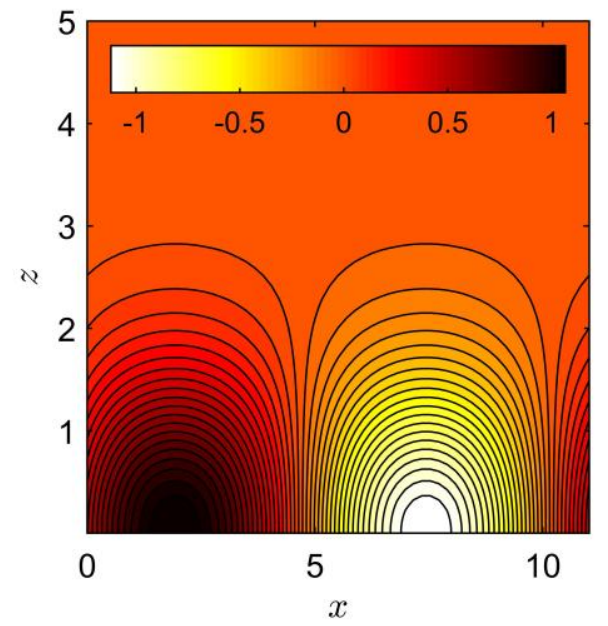

Fig. 17. Fastest-growing disturbance mode: one-dimensional eigenvectors a) vorticity, b) stream function and two-dimensional eigenfunctions c) vorticity, d) stream function $\left(\boldsymbol{N}=\mathbf{8 0}, \boldsymbol{\tau}_{\boldsymbol{s}}=\mathbf{1 2 6}\right)$

\section{Conclusions}

In this study, an idealized mathematical model has been formulated to investigate whether the wavy interfacial morphology in metals joined in HVIW could be the signature of a sheardriven high strain-rate linear instability mechanism. A differential eigenvalue problem was obtained by linearizing Cauchy's equations about a representative perfectly-plastic shear flow and substituting a normal mode decomposition of the perturbation fields corresponding to a 
disturbance varying sinusoidally in the coordinate direction parallel to the weld. A Chebyshev spectral collocation scheme was used to discretize the differential eigenvalue problem in the (z) coordinate transverse to the weld. The resulting algebraic eigensystem was solved numerically, yielding the growth rate and phase speed of the fastest-growing instability modes as functions of the mode wavenumber $k$ and system control parameters, particularly the dimensionless shear yield stress $\tau_{s}$.

Relative to prior stability analyses of this phenomenon, novel features of the present study include the incorporation of a perfectly-plastic rather than Newtonian viscous or inviscid (i.e., strictly fluid) constitutive model and a jet-like (symmetric) rather than shear-layer-like (antisymmetric) base flow profile. The former specification is motivated by the fact that joining in HVIW occurs without melting, while the latter accords with interfacial deformation profiles of HVIW processes simulated numerically using finite-element analysis. Together, these specifications lead to a singularity in the differential eigenvalue problem at the mean position of the interface between the two plates. Although the disturbance behavior is shown to be regular there, customized boundary conditions are derived to avoid numerical ill-conditioning.

The results computed here suggest that an instability that gives rise to a wavy morphology is possible, even with the minimal set of physical ingredients included; that is, melting is not necessary. Specifically, using a dimensional jet thickness $\widetilde{\beta}^{-1}$ of $\approx 15-20 \mu m$ based on finite-element simulations [1], the dimensional wavelength of the fastest-growing disturbance for the scenario with $\tau_{s}=0.0126$ (corresponding to a physically-realistic maximum jet speed near the collision point of about $1000 \mathrm{~m} / \mathrm{s}$ ) is approximately $\tilde{\lambda}=$ $\left(\frac{2 \pi}{k}\right) \tilde{\beta}^{-1}=\left(\frac{2 \pi}{0.75}\right) 15 \mu m=125 \mu m$, in good agreement with simulation results [32]. Note that the result that the fastest-growing instability mode has a streamwise wavelength comparable to the shear layer thickness commonly arises in fluid dynamical contexts (i.e., for inviscid or large Reynolds number Newtonian flows) [33]. Moreover, the dimensional time scale $\left(\tilde{\beta} \tilde{u}_{0}\right)^{-1} \sigma_{r}{ }^{-1}$ for the amplitude of the disturbance to grow significantly is $0.2 \mu s$, much less than the (short) time scale $\approx 70 \mu \mathrm{s}$ for the overall process. This suggests that the frozen-coefficient (quasi-static) analyses employed here is reasonable, and confirms the potential physical relevance of the proposed instability mechanism.

Of course, the analysis presented here constitutes only a first step in the quantitative stability investigation of the mechanics underlying wavy pattern formation in HVIW. Important 
next steps include employing a non-modal approach that not only allows for a time-dependent but also $x$-varying (i.e., in the direction parallel to the weld) base flow and including nonlinearities, which are required to determine the amplitude of the emergent wavy patterns. These and other extensions await future work.

\section{Acknowledgments}

Funding from the U.S. National Science Foundation (CMII-0928319) is gratefully acknowledged.

\section{References}

[1] A. Nassiri, G. Chini, A. Vivek, G. Daehn, B. Kinsey, Arbitrary Lagrangian-Eulerian finite element simulation and experimental investigation of wavy interfacial morphology during high velocity impact welding, Materials \& Design 88 (2015) 245-358.

[2] Y. Zhang, S. S. Babu, C. Prothe, M. Blakely, J. Kwasegroch, M. LaHa, , G. S. Daehn, Application of high velocity impact welding at varied different length scales, Journal of Materials Processing Technology 211(5) (2011) 944-952.

[3] J. Lueg-Althoff, A. Lorenz, S. Gies, C. Weddeling, G. Goebel, A. E. Tekkaya, E. Beyer, Magnetic Pulse Welding by Electromagnetic Compression: Determination of the Impact Velocity, Advanced Materials Research 966-967 (2014) 489-499.

[4] J.Verstraete, W. De Waele, K. Faes, Magnetic pulse welding: lessons to be learned from explosive welding. Sustainable Construction and Design 2(3) (2011) 458-464.

[5] A. Nassiri, C. Campbell, G. Chini, B. Kinsey, Analytical model and experimental validation of single turn, axi-symmetric coil for electromagnetic forming and welding, Procedia Manufacturing 1 (2015) 814-827

[6] R. Mendes, J. B. Ribeiro, A. Loureiro, Effect of explosive characteristics on the explosive welding of stainless steel to carbon steel in cylindrical configuration, Materials \& Design 51 (2013) 182-192.

[7] K. Faes, T. Baaten, W. De Waele, N. Debroux, Joining of Copper to Brass using magnetic pulse welding, Proceedings of the 4th International conference on High Speed Forming, Columbus, OH, (2010) 84-96.

[8] A. Vivek, G. Taber, D. Demmerle, Glenn Daehn, Effect of interchanging flyer sheet and target plate material on interface morphology during laser impact welding, (2015), Unpublished manuscript.

[9] A. Vivek, S. R. Hansen, B.C. Liu, G. S. Daehn, Vaporizing foil actuator: A tool for collision welding. Journal of Materials Processing Technology 213 (12) (2013) 2304-2311.

[10] S. R. Reid, A discussion of the mechanism of interface wave generation in explosive welding, International Journal of Mechanical Sciences 16(6) (1974) 399-413. 
[11] G. R. Abrahamson, Permanent periodic surface deformations due to a traveling jet, Journal of applied mechanics, 28(4) (1961) 519-528.

[12] A. S. Bahrani, T. J. Black, B. Crossland, The mechanics of wave formation in explosive welding. Proceedings of the Royal Society of London. Series A. Mathematical and Physical Sciences, (1967) 296(1445), 123-136.

[13] H. El-Sobky, T. Z. Blazynski, Experimental investigation of the mechanics of explosive welding by means of a liquid analogue. Procceeding of 5th International Conference on "High Energy Rate fabrication" (1975) 1-21, Denver, Colorado.

[14] G. R. Cowan, O. R. Bergmann, A. H. Holtzman, Mechanism of bond zone wave formation in explosion-clad metals. Metallurgical and Materials Transactions B (1971) 2(11), 3145-3155.

[15] J. N. Hunt, Wave formation in explosive welding, Philosophical magazine, 17(148) (1968) 669-680.

[16] M. P. W. Wilson, J. H. Brunton, Wave formation between impacting liquids in explosive welding and erosion. Nature 226 (1970) 538-541.

[17] J. L. Robinson, The mechanics of wave formation in impact welding, Philosophical Magazine, 31(3) (1975) 587-597.

[18] J. F. Kowalick, D. R. Hay, A mechanism of explosive bonding, Metallurgical Transactions, 2(7) (1971) 1953-1958.

[19] E. P. Carton, Wave forming mechanisms in explosive welding, Materials Science Forum, 465 (2004) 219224.

[20] A. A. Mousavi, S. T. S. Al-Hassani, Numerical and experimental studies of the mechanism of the wavy interface formations in explosive/impact welding, Journal of the Mechanics and Physics of Solids, 53(11) (2005) 2501-2528.

[21] A. Nassiri, G. Chini, B. Kinsey, Spatial stability analysis of emergent wavy interfacial patterns in magnetic pulsed welding, CIRP Annals-Manufacturing Technology, 63(1) (2014) 245-248.

[22] A. Ben-Artzy, A. Stern, N. Frage, V. Shribman, Interface phenomena in aluminium-magnesium magnetic pulse welding, Science and Technology of Welding \& Joining, 13(4) (2008) 402-408.

[23] C. Chemin, C., T. Qingming, Mechanism of wave formation at the interface in explosive welding, Acta Mechanica Sinica, 5(2) (1989) 97-108.

[24] L. M. Kachanov, Fundamentals of the Theory of Plasticity, 2004, Courier Corporation.

[25] L.N. Trefethen, Spectral Methods in MATLAB, SIAM, 2000, Philadelphia, PA.

[26] J.P. Boyd, Chebyshev and Fourier Spectral Methods, 2001, New York: Dover Publication.

[27] L.F. Shampine, I. Gladwell, S. Thompson, Solving ODEs with MATLAB, 2003, Cambridge University Press.

[28] R. H. Wittman, The influence of collision parameters on the strength and microstructure of an explosion welded aluminum alloy. Proc. 2nd Sym. on Use of Explosive Energy in Manufacturing Metallic Materials of New Properties and Possibilities of Application thereof in the Chemical Industry, 1973, 153-168. 
[29] A. A. Deribas, V. A. Simonov, I. D. Zakcharenko, Investigation of explosive welding parameters for arbitrary combinations of metals and alloys. Proc. 5th Int. Conf. on High Energy Rate Fabrication, 4, University of Denver, Denver, C0 1975, 1-4

[30] D. Jaramillo, A. Szecket, O. T. Inal, On the transition from a waveless to a wavy Interface in explosive welding. Materials Science and Engineering, 91 (1987) 217-222.

[31] A. Nassiri, G. P. Chini, B. L. Kinsey, Arbitrary Lagrangian Eulerian FEA Method to Predict Wavy Pattern and Weldability Window During Magnetic Pulsed Welding, In ASME 2015 International Manufacturing Science and Engineering Conference (2015), (pp. V001T02A100-V001T02A100), American Society of Mechanical Engineers, Charlotte, NC.

[32] A. Nassiri, Investigation of wavy interfacial morphology in magnetic pulsed welding: mathematical modeling, numerical simulations and experimental tests, University of New Hampshire, 2015, 167 pages; AAT 3708454.

[33] P.G. Drazin, Introduction to Hydrodynamic Stability, 2002, Cambridge University Press.

[34] W. O. Criminale, T. L. Jackson, R.D. Joslin, Theory and Computation in Hydrodynamic Stability, 2003, Cambridge University Press, Cambridge, UK. 


\section{APPENDIX}

In this appendix, a local analysis of the ordinary differential eigensystem (49)-(50) as $z \rightarrow 0^{+}$is performed. Substituting Eq. (49) into Eq. (50) yields

$$
\left[\left(\sigma+i k U_{B}\right)+\frac{\alpha}{|s|}\right] D^{2} \hat{\psi}+\alpha D\left[\frac{1}{|s|}\right] \cdot D \hat{\psi}-\left[k^{2}\left(\sigma+i k U_{B}\right)+(i k) \frac{d^{2} U_{B}}{d z^{2}}\right] \widehat{\psi}=0
$$

where $S \equiv \frac{d U_{B}}{d z}, \mathrm{D}=\frac{d}{d z}$, and $\alpha=4 \tau_{s} k^{2}$. To examine the solution behavior near $z=0$, the jet base-flow profile and its $z$-derivatives are expanded in Taylor series there. Thus,

$$
U_{B}=e^{-\frac{z^{2}}{2}} \sim 1-\frac{z^{2}}{2}+\frac{z^{4}}{8}-\cdots
$$

The first derivative of the base flow can be expressed as

$$
\begin{gathered}
\frac{d U_{B}}{d z}=-z U_{B} \sim-z\left(1-\frac{z^{2}}{2}+\frac{z^{4}}{8}-\cdots\right) \\
|S| \equiv\left|\frac{d U_{B}}{d z}\right|=z\left(1-\frac{z^{2}}{2}+\frac{z^{4}}{8}-\cdots\right) \text { for } 0 \leq z \ll 1
\end{gathered}
$$

The second derivative of the base flow

$$
\frac{d^{2} U_{B}}{d z^{2}}=-U_{B}+z^{2} U_{B} \sim-1+\frac{3}{2} z^{2}-\frac{5}{8} z^{4}+\cdots
$$

Consider the term $\alpha \mathrm{D}\left[\frac{1}{|S|} D \hat{\psi}\right]$ in Eq. (50) and Eq. (A.1):

$$
\alpha \mathrm{D}\left[\frac{1}{|S|} D \hat{\psi}\right]=\frac{\alpha}{|S|} D^{2} \hat{\psi}+\alpha \mathrm{D}\left[\frac{1}{|S|}\right] \cdot D \hat{\psi}
$$

For $z>0$

$$
\begin{aligned}
\mathrm{D}\left[\frac{1}{|S|}\right]=D\left[\frac{1}{z U_{B}}\right] & =e^{\frac{z^{2}}{2}}\left(1-z^{-2}\right) \sim\left(1+\frac{z^{2}}{2}+\frac{z^{4}}{8}+\cdots\right)\left(1-z^{-2}\right) \\
& \sim-\frac{1}{z^{2}}+\frac{1}{2}+\frac{3}{8} z^{2}+\frac{5}{48} z^{4}+\cdots
\end{aligned}
$$

Finally,

$$
\frac{1}{|S|}=\frac{1}{z U_{B}}=\frac{e^{\frac{z^{2}}{2}}}{z} \sim \frac{1}{z}+\frac{z}{2}+\frac{z^{3}}{8}+\frac{z^{5}}{48}+\cdots \quad \text { for } z>0
$$

Thus, as $z \rightarrow 0^{+}$Eq. (A.1) may be approximated as 


$$
\begin{aligned}
{\left[\frac{\alpha}{z}+(\sigma+i k)\right.} & \left.+\frac{\alpha z}{2}+\frac{(-i k) z^{2}}{2}+\frac{\alpha z^{3}}{8}+\frac{(i k) z^{4}}{8}\right] D^{2} \widehat{\psi}+\left[-\frac{\alpha}{z^{2}}+\frac{\alpha}{2}+\frac{3 \alpha}{8} z^{2}\right] D \widehat{\psi} \\
& -\left[k^{2}(\sigma+i k)-i k-\frac{i k^{3} z^{2}}{2}+\frac{3(i k) z^{2}}{2}+\frac{i k^{3} z^{4}}{8}-\frac{5(i k) z^{4}}{8}\right] \widehat{\psi} \approx 0
\end{aligned}
$$

From Eq. (A.9) it is readily concluded that $z=0$ is regular singular point (or nonessential singularity) of Eq. (A.1). Thus, the solution behavior can be determined using the method of Frobenius. Specifically, a local approximation as $z \rightarrow 0^{+}$for $\hat{\psi}$ is posited having the following form:

$$
\hat{\psi} \sim z^{r} \sum_{n=0}^{\infty} \psi_{n} z^{n} \sim z^{r}\left(\psi_{0}+\psi_{1} z+\psi_{2} z^{2}+\cdots\right)
$$

where the indicial index $r$ is to be determined from the analysis. The first and second derivatives of $\hat{\psi}$ thus are

$$
\begin{gathered}
D \hat{\psi} \sim \sum_{n=0}^{\infty}(n+r) \psi_{n} z^{(n+r-1)} \\
D^{2} \hat{\psi} \sim \sum_{n=0}^{\infty}(n+r)(n+r-1) \psi_{n} z^{(n+r-2)}
\end{gathered}
$$

Substituting Eqs. (A.10)-(A.12) into Eq. (A.9) and rearranging and relabeling indices yields

$$
\begin{aligned}
& \sum_{n=0}^{\infty}\left[\alpha(n+r)(n+r-2) \psi_{n}\right] z^{(n+r-3)} \\
& +\sum_{n=1}^{\infty}\left[(\sigma+i k)(n+r-1)(n+r-2) \psi_{n-1}\right] z^{(n+r-3)} \\
& +\sum_{n=2}^{\infty}\left[\frac{\alpha}{2}(n+r-2)^{2} \psi_{n-2}\right] z^{(n+r-3)} \\
& +\sum_{n=3}^{\infty}\left\{\left[\left(-\frac{i k}{2}\right)(n+r-3)(n+r-4)-\left(k^{2}(\sigma+i k)-i k\right)\right] \psi_{n-3}\right\} z^{(n+r-3)} \\
& +\sum_{n=4}^{\infty}\left[\frac{\alpha}{8}(n+r-2)(n+r-4) \psi_{n-4}\right] z^{(n+r-3)}
\end{aligned}
$$




$$
+\sum_{n=5}^{\infty}\left\{\left[\left(\frac{i k}{8}\right)(n+r-5)(n+r-6)+\frac{i k^{3}}{2}\right] \psi_{n-5}\right\} z^{(n+r+2)}+\cdots=0
$$

Collecting terms when $n=0$ requires

$$
r(r-2) \psi_{0}=0 \Rightarrow r=0,2
$$

Collecting terms when $n=1$ gives

$$
\alpha(r+1)(r-1) \psi_{1}=-(\sigma+i k)(r)(r-1) \psi_{0}
$$

and, since $r \neq 1$,

$$
\psi_{1}=-\left(\frac{\sigma+i k}{\alpha}\right)\left(\frac{r}{r+1}\right) \psi_{0}
$$

When $n=2$,

$$
[\alpha(r+2) r] \psi_{2}+[(\sigma+i k)(r+1) r] \psi_{1}+\frac{\alpha}{2} r^{2} \psi_{0}=0
$$

Note that for $r=0$, Eq. (A.17) places no constraint on $\psi_{2}$, while for $r \neq 0$,

$$
\psi_{2}=-\left(\frac{\sigma+i k}{\alpha}\right)\left(\frac{r+1}{r+2}\right) \psi_{1}-\frac{1}{2}\left(\frac{r}{r+2}\right) \psi_{0}
$$

Substituting the expression for $\psi_{1}$ from Eq. (A.16) into Eq. (A.18) yields

$$
\psi_{2}=\left[\left(\frac{\sigma+i k}{\alpha}\right)^{2}-\frac{1}{2}\right]\left(\frac{r}{r+2}\right) \psi_{0}
$$

When $n=3$,

$$
\begin{gathered}
{[\alpha(r+3)(r+1)] \psi_{3}+[(\sigma+i k)(r+2)(r+1)] \psi_{2}+\frac{\alpha}{2}(r+1)^{2} \psi_{1}} \\
=\left[\frac{i k}{2}(r)(r-1)+k^{2}(\sigma+i k)-i k\right] \psi_{0}
\end{gathered}
$$

Rearranging Eq. (A.20) yields

$$
\psi_{3}=-\left(\frac{\sigma+i k}{\alpha}\right)\left(\frac{r+2}{r+3}\right) \psi_{2}-\frac{1}{2}\left(\frac{r+1}{r+3}\right) \psi_{1}+\frac{\left[\frac{i k}{2} r(r-1)+k^{2}(\sigma+i k)-i k\right]}{\alpha(r+3)(r+1)} \psi_{0}
$$

Substituting gives

$$
\begin{aligned}
-\frac{1}{2}\left(\frac{r+1}{r+3}\right) \psi_{1} & =\left[-\frac{1}{2}\left(\frac{r+1}{r+3}\right)\right]\left[\left(-\frac{r}{r+1}\right)\left(\frac{\sigma+i k}{\alpha}\right)\right] \psi_{0} \\
& =\frac{1}{2}\left(\frac{\sigma+i k}{\alpha}\right)\left(\frac{r}{r+3}\right) \psi_{0}
\end{aligned}
$$

and, for $r \neq 0$, 


$$
\begin{gathered}
-\left(\frac{\sigma+i k}{\alpha}\right)\left(\frac{r+2}{r+3}\right) \psi_{2}=-\left(\frac{\sigma+i k}{\alpha}\right)\left(\frac{r+2}{r+3}\right)\left[\left(\frac{\sigma+i k}{\alpha}\right)^{2}-\frac{1}{2}\right]\left(\frac{r}{r+2}\right) \psi_{0} \\
=\left[-\left(\frac{\sigma+i k}{\alpha}\right)^{3}+\frac{1}{2}\left(\frac{\sigma+i k}{\alpha}\right)\right]\left(\frac{r}{r+3}\right) \psi_{0}
\end{gathered}
$$

Collecting the results,

$$
\begin{array}{cc}
r=0: & \psi_{3}=-\left(\frac{2}{3}\right)\left(\frac{\sigma+i k}{\alpha}\right) \psi_{2}+\left(\frac{k^{2}(\sigma+i k)-i k}{3 \alpha}\right) \psi_{0} \\
r=2: & \psi_{3}=\left(\frac{2}{5}\right)\left(\frac{\sigma+i k}{\alpha}\right)\left[1-\left(\frac{\sigma+i k}{\alpha}\right)^{2}\right] \psi_{0}+\left(\frac{k^{2}(\sigma+i k)}{15 \alpha}\right) \psi_{0}
\end{array}
$$

Finally, when $n=4$,

$$
\begin{aligned}
{[\alpha(r+4)(r+2)] \psi_{4}+[(\sigma+i k)(r+3)(r+2)] \psi_{3}+\frac{\alpha}{2}(r+2)^{2} \psi_{1} } \\
=\left[\frac{i k}{2}(r+1)(r)+\left(k^{2}(\sigma+i k)-i k\right)\right] \psi_{0}-\frac{\alpha}{2}(r+2)(r) \psi_{0}
\end{aligned}
$$

and solving for $\psi_{4}$ yields

$$
\begin{aligned}
\psi_{4}=-\left(\frac{\sigma+i k}{\alpha}\right) & \left(\frac{r+3}{r+4}\right) \psi_{3}-\frac{1}{2}\left(\frac{r+2}{r+4}\right) \psi_{2} \\
+ & \frac{\left[\frac{i k}{2}(r+1)(r)+k^{2}(\sigma+i k)-i k\right]}{\alpha(r+4)(r+2)} \psi_{1}-\frac{1}{8}\left(\frac{r}{r+8}\right) \psi_{0}
\end{aligned}
$$

When $r=0$,

$$
\psi_{4}=-\left(\frac{3}{4}\right)\left(\frac{\sigma+i k}{\alpha}\right) \psi_{3}-\frac{1}{4} \psi_{2}+\left(\frac{k^{2}(\sigma+i k)-i k}{8 \alpha}\right) \psi_{1}
$$

Upon substituting for $\psi_{3}$ and $\psi_{1}$, Eq. (A.28) can be further reduced.

$$
r=0: \quad \psi_{4}=\frac{1}{2}\left[\left(\frac{\sigma+i k}{\alpha}\right)^{2}-\frac{1}{2}\right] \psi_{2}-\left(\frac{\sigma+i k}{\alpha}\right)\left(\frac{k^{2}(\sigma+i k)-i k}{4 \alpha}\right) \psi_{0}
$$

The local behavior of $\hat{\psi}$ as $z \rightarrow 0^{+}$can now be reconstructed. Recalling expansion Eq. (A.10), the approximate solutions $\hat{\psi}^{(0)}$ and $\hat{\psi}^{(2)}$ for $r=0$ and $r=2$, respectively, are:

$$
\begin{gathered}
\hat{\psi}^{(0)} \sim a\left[1+\left(\frac{k^{2}(\sigma+i k)-i k}{3 \alpha}\right) z^{3}-\left(\frac{\sigma+i k}{\alpha}\right)\left(\frac{k^{2}(\sigma+i k)-i k}{4 \alpha}\right) z^{4}\right] \\
+b\left[z^{2}-\frac{2}{3}\left(\frac{\sigma+i k}{\alpha}\right) z^{3}+\left(\frac{1}{2}\left(\frac{\sigma+i k}{\alpha}\right)^{2}-\frac{1}{4}\right) z^{4}\right] \\
\hat{\psi}^{(2)} \sim c\left[z^{2}-\left(\frac{2}{3}\right)\left(\frac{\sigma+i k}{\alpha}\right) z^{3}+\left(\frac{1}{2}\left(\frac{\sigma+i k}{\alpha}\right)^{2}-\frac{1}{4}\right) z^{4}\right]
\end{gathered}
$$


where in Eq. (A.30) the free constants $\psi_{0}$ and $\psi_{2}$ have been relabeled $a$ and $b$, respectively, and in Eq. (A.31) the free constant $\psi_{0}$ has been relabeled $c$. Note that these solutions are linearly independent and, without loss of generality, the free constant $b$ arising in $\hat{\psi}^{(0)}$ can be set to zero (since the term it multiplies is a multiple of $\hat{\psi}^{(2)}$ ).

By taking appropriate linear combinations of $\hat{\psi}^{(0)}$ and $\widehat{\psi}^{(2)}$, two families of solutions can be formed that are distinguished by the value of $\hat{\psi}$ at $z=0$. Specifically, "even" and "odd" modes can be identified such that for the even mode $\hat{\psi} \neq 0$ at $z=0$, while for the odd mode $\hat{\psi}=0$ at $z=0$. Thus, for the even mode,

$$
\widehat{\psi}^{(e)} \sim a\left[1+\left(\frac{k^{2}(\sigma+i k)-i k}{3 \alpha}\right) z^{3}+\cdots\right]+c\left[z^{2}-\frac{2}{3}\left(\frac{\sigma+i k}{\alpha}\right) z^{3}+\cdots\right]
$$

$c$ can be chosen to cancel the $z^{3}$ term; i.e.,

$$
c=\left(\frac{k^{2}(\sigma+i k)-i k}{3 \alpha}\right)\left(\frac{3 \alpha}{2(\sigma+i k)}\right) a=\frac{1}{2}\left[k^{2}-\frac{i k}{\sigma+i k}\right] a
$$

Hence,

$$
\hat{\psi}^{(e)}=a\left[1+\left(\frac{k^{2}}{2}-\frac{1}{2} \frac{i k}{\sigma+i k}\right) z^{2}+O\left(z^{4}\right)\right]
$$

For the odd mode, the bare $\hat{\psi}^{(o)}$ may be used:

$$
\widehat{\psi}^{(o)} \sim c\left[z^{2}-\frac{2}{3}\left(\frac{\sigma+i k}{\alpha}\right) z^{3}+O\left(z^{4}\right)\right]
$$

In this investigation, however, only the even mode is considered, primarily because very many related stability problems the even mode is known to be the fastest-growing mode [34] .

Although $\hat{\psi}$ is smooth as $z \rightarrow 0^{+}$(i.e., there is not a logarithmic singularity in $\hat{\psi}$ although the two indicial exponents, $r=0,2$, differ by an integer), to avoid division by zero the single boundary condition $\frac{d \hat{\psi}}{d z}=0$ at $z=0$ is replaced by two effective boundary conditions at $z=\delta \ll 1$. To derive these boundary conditions, Eq. (A.32) for the even mode is used: 


$$
\widehat{\psi}(\delta)=a+a\left(\frac{k^{2}}{2} \delta^{2}\right)-\frac{a}{2}\left(\frac{i k}{\sigma+i k}\right) \delta^{2}
$$

For a linear eigenvalue problem, it is necessary to re-arrange Eq. (A.36):

$$
(i k) \hat{\psi}(\delta)+\left[\left(\frac{i k}{2}\right) \delta^{2}\left(1-k^{2}\right)-i k\right] a=-\sigma \widehat{\psi}(\delta)+\sigma\left(1+\frac{k^{2} \delta^{2}}{2}\right) a
$$

The introduction of the a priori unknown parameter $a$ requires the specification of an additional boundary condition at $z=\delta$. For this purpose, note that

$$
D \hat{\psi}(\delta)=a\left[k^{2}-\left(\frac{i k}{\sigma+i k}\right)\right] \delta
$$

Rearranging Eq. (A.38) gives

$$
(i k) D \widehat{\psi}(\delta)+i(k \delta)\left[1-k^{2}\right] a=-\sigma D \hat{\psi}(\delta)+\sigma\left(\delta k^{2}\right) a
$$

Thus, Eqs. (A.37) and (A.39) are used in lieu of the original condition $\frac{\partial \psi}{\partial z}=0$ at $z=0$ to ameliorate the numerical ill-conditioning associated with imposition of the latter constraint. 